\title{
DIRECT DETECTION OF DARK MATTER RATES FOR VARIOUS WIMPS
}

\author{
V.K. Oikonomou ${ }^{1}$, J.D. Vergados ${ }^{2 *}$ and Ch.C. Moustakidis ${ }^{1}$ \\ ${ }^{1}$ Department of Theoretical Physics, Aristotle University of Thessaloniki, 54124 Thessaloniki, Greece \\ 2 University of Ioannina, Ioannina, GR 45110, Greece.
}

\begin{abstract}
The event rates for the direct detection of dark matter candidates, originating from UED scenario, are evaluated for a number of nuclear targets. Realistic form factors as well as spin ME and response functions are employed. Due to LR+RL helicities contribution, the proton amplitude is found to be dominant. Various other non-susy dark matter candidates are examined at the end.
\end{abstract}

PACS numbers: $95.35+\mathrm{d}, 12.60 . \mathrm{Jv}$.

\section{INTRODUCTION.}

Models with compact extra dimensions offer rich and interesting phenomenology [1, 2, 3, 4, 5, 6, 7, [8]. In such models fields propagating in extra dimensions at low energies appear as a tower of massive particles corresponding to a given charge and spin. The massive states are nothing but modes of the fields carrying quantized momentum in extra dimensions. This means that the spacing of the towers is $\frac{1}{R}$, i.e. the inverse of the characteristic size in extra dimensions. In this scheme the ordinary particles are associated with the zero modes. In brane world models only fields interacting gravitationally can propagate in extra dimensions, i.e. the excitations are of the Kaluza-Klein (K-K) type. Models with Universal Extra Dimensions can have stable particles, due to KK parity, originating from higher dimensional Poincare invariance [6]. Under this parity the even modes, including the ordinary particles, are even and the odd modes are odd. Thus the lightest odd mode particle is cosmologically stable. For a recent review we refer the reader to the literature 9]. Like the neutralino, it must be a neutral a weakly interacting particle. It can thus serve as a viable dark matter candidate, which, together with dark energy, seems to dominate in the Universe $10,11,12,13,14,15,16$.

The K-K WIMP's (Weekly Interacting Massive Particles) velocity dependence can be assumed to be the same with that used in neutralino calculations, since a particle's rotational velocity is independent of its mass. The kinematics involved is, by and large, similar to that of the neutralino, leading to cross sections which are proportional $\mu_{r}$, the WIMP-nucleus reduced mass. Furthermore the nuclear physics input, which depends on $\mu_{r} \simeq A m_{p}$, is expected to be the same. There are appear two differences compared to the neutralino, though, both related to its larger mass.

- The density (number of particles per unit volume) of a WIMP falls inversely proportional to its mass. Thus, since in K-K theories the WIMP mass is much larger than that of the target, for a given WIMP-nucleon gross section, the event rate is inversely proportional to the WIMP mass. This means that the limits on the nucleon cross section extracted from the data must be rising with the square root of the WIMP mass. This allows for nucleon cross sections larger than those extracted for the neutralino.

- The average neutralino energy is now quite higher. In fact for a Maxwell-Boltzmann (M-B) velocity distribution one finds that $\left\langle T_{W I M P}\right\rangle=\frac{3}{4} M_{W I M P} v_{0}^{2}$, with $v_{0}$ the characteristic velocity of the M-B distribution, which coincides with the sun's rotational velocity, $v_{0} \simeq$ $2.2 \times 10^{5} \mathrm{~km} / \mathrm{s}$. Thus one finds

$$
<T_{W I M P}>\simeq 40\left(\frac{m_{W I M P}}{100 \mathrm{GeV}}\right) \mathrm{keV} .
$$

* e-mail: vergados@cc.uoi.gr 
Furthermore, since the maximum allowed velocity is $v_{e s c}=2.84 v_{0}$, we find that

$$
T_{\max } \simeq 120\left(\frac{m_{W I M P}}{100 \mathrm{GeV}}\right) \mathrm{keV} .
$$

Thus for a K-K WIMP with mass $1 \mathrm{TeV}$, the average WIMP energy is $0.4 \mathrm{MeV}$ and the maximum energy is $1.2 \mathrm{MeV}$. Thus in this case, due to the high velocity tail of the velocity distribution it is reasonable to expect an energy transfer to the nucleus in the MeV region. So one need not attempt to detect such a heavy WIMP the hard way, i.e. by measuring the energy of the recoiling nucleus, as in the case of the neutralino. Many nuclear targets can now be excited by the WIMP-nucleus interaction and the de-excitation photons can be easily detected.

\section{THE KALUZA-KLEIN BOSON AS A DARK MATTER CANDIDATE}

Assuming small boundary terms we expect that the lightest exotic particle, which can serve as a dark matter candidate, is a gauge boson $B^{1}$ having the same quantum numbers and couplings with the Standard Model gauge boson $B$, except that it has K-K parity -1 . Thus its couplings involve another negative K-K parity particle. In this work we will assume that such particles are the K-K quarks, partners of the ordinary quarks, but much heavier [1, 2].

\section{Intermediate K-K quarks}

In this case a generic Feynman diagrams are shown in Fig. 1, The corresponding amplitude

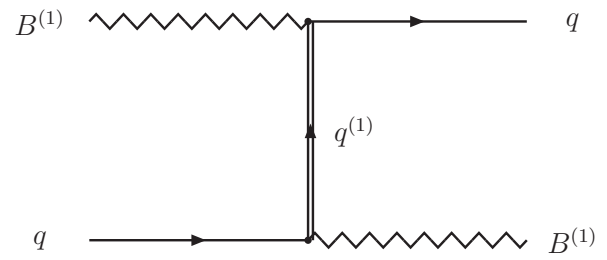

(a)

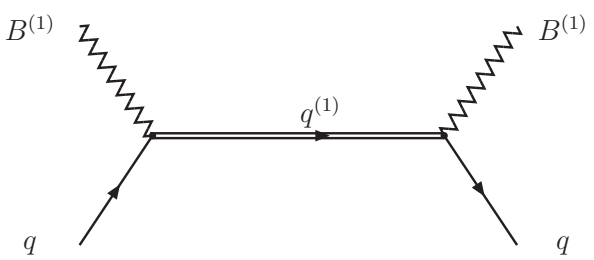

(b)

FIG. 1: Two diagrams leading to the interaction of $\mathrm{K}-\mathrm{K}$ gauge boson $B^{1}$ with quarks at tree level mediated by $\mathrm{K}-\mathrm{K}$ quarks

involving left-handed quarks is given by:

$$
\begin{aligned}
& \mathcal{M}_{1}^{L}(q)=-i\left(g_{1} Y_{L}\right)^{2}\left[\overline{q_{L}}(x) \gamma^{\nu} \frac{\left(\not p_{q}-\not p_{B^{\prime}}+m_{q^{(1)}}\right)}{\left(p_{q}-p_{B^{\prime}}\right)^{2}-m_{q^{(1)}}^{2}} \gamma^{\mu} q_{L}(x)\right] \epsilon_{\mu}^{*}\left(p_{B}^{\prime}\right) \epsilon_{\nu}\left(p_{B}\right), \\
& \mathcal{M}_{2}^{L}(q)=-i\left(g_{1} Y_{L}\right)^{2}\left[\overline{q_{L}}(x) \gamma^{\mu} \frac{\left(\not p_{q}+\not p_{B}+m_{\left.q^{(1)}\right)}\right)}{\left(p_{q}+p_{B}\right)^{2}-m_{q^{(1)}}^{2}} \gamma^{\nu} q_{L}(x)\right] \epsilon_{\mu}^{*}\left(p_{B^{\prime}}\right) \epsilon_{\nu}\left(p_{B}\right),
\end{aligned}
$$

where $g_{1}=g \tan \theta_{W}=\sqrt{4 \sqrt{2} G_{F}} m_{W} \tan \theta_{W}, Y_{L}=1 / 3$ and $\epsilon_{\mu}\left(p_{B}^{\prime}\right), \epsilon_{\nu}\left(p_{B}\right)$ are the helicities of the $\mathrm{K}-\mathrm{K}$ bosons. For the right handed quarks of the upper type we have,

$$
\begin{aligned}
& \mathcal{M}_{1}^{R}(u)=-i\left(g_{1} 4 / 3\right)^{2}\left[\overline{u_{R}}(x) \gamma^{\nu} \frac{\left(\not p_{q}-\not p_{B^{\prime}}\right)}{\left(p_{q}-p_{B^{\prime}}\right)^{2}-m_{q^{(1)}}^{2}} \gamma^{\mu} u_{R}(x)\right] \epsilon_{\mu}^{*}\left(p_{B}^{\prime}\right) \epsilon_{\nu}\left(p_{B}\right) \\
& \mathcal{M}_{2}^{R}(u)=-i\left(g_{1} 4 / 3\right)^{2}\left[\overline{u_{R}}(x) \gamma^{\mu} \frac{\left(\not p_{q}+\not p_{B}\right)}{\left(p_{q}+p_{B}\right)^{2}-m_{q^{(1)}}^{2}} \gamma^{\nu} u_{R}(x)\right] \epsilon_{\mu}^{*}\left(p_{B^{\prime}}\right) \epsilon_{\nu}\left(p_{B}\right) .
\end{aligned}
$$


For the right handed down quarks we have,

$$
\begin{aligned}
& \mathcal{M}_{1}^{R}(d)=-i\left(g_{1}(-2 / 3)\right)^{2}\left[\overline{d_{R}}(x) \gamma^{\nu} \frac{\left(\not p_{q}-\not p_{B^{\prime}}\right)}{\left(p_{q}-p_{B^{\prime}}\right)^{2}-m_{q^{(1)}}^{2}} \gamma^{\mu} d_{R}(x)\right] \epsilon_{\mu}^{*}\left(p_{B}^{\prime}\right) \epsilon_{\nu}\left(p_{B}\right), \\
& \mathcal{M}_{2}^{R}(d)=-i\left(g_{1}(-2 / 3)\right)^{2}\left[\overline{u_{R}}(x) \gamma^{\mu} \frac{\left(\not p_{q}+\not p_{B}\right)}{\left(p_{q}+p_{B}\right)^{2}-m_{q^{(1)}}^{2}} \gamma^{\nu} d_{R}(x)\right] \epsilon_{\mu}^{*}\left(p_{B^{\prime}}\right) \epsilon_{\nu}\left(p_{B}\right) .
\end{aligned}
$$

We also have R-L interference terms, which seem to have been missed in the previous calculations [1, 2]. One finds:

$$
\begin{aligned}
\mathcal{M}_{1}^{L R}(u) & =-i\left(g_{1}\right)^{2} 4 / 9\left[\overline{u_{L}}(x) \gamma^{\nu} \frac{m_{q^{(1)}}}{\left(p_{q}-p_{B^{\prime}}\right)^{2}-m_{q^{(1)}}^{2}} \gamma^{\mu} u_{R}(x)+H . C .\right] \epsilon_{\mu}^{*}\left(p_{B}^{\prime}\right) \epsilon_{\nu}\left(p_{B}\right), \\
\mathcal{M}_{2}^{L R}(u) & =-i\left(g_{1}\right)^{2} 4 / 9\left[\overline{u_{L}}(x) \gamma^{\mu} \frac{m_{q^{(1)}}}{\left(p_{q}+p_{B}\right)^{2}-m_{q^{(1)}}^{2}} \gamma^{\nu} u_{R}(x)+H . C .\right] \epsilon_{\mu}^{*}\left(p_{B^{\prime}}\right) \epsilon_{\nu}\left(p_{B}\right), \\
\mathcal{M}_{1}^{L R}(d) & =-i\left(g_{1}\right)^{2}(-2 / 9)\left[\overline{d_{L}}(x) \gamma^{\nu} \frac{m_{q^{(1)}}}{\left(p_{q}-p_{B^{\prime}}\right)^{2}-m_{q^{(1)}}^{2}} \gamma^{\mu} d_{R}(x)+H . C .\right] \epsilon_{\mu}^{*}\left(p_{B}^{\prime}\right) \epsilon_{\nu}\left(p_{B}\right), \\
\mathcal{M}_{2}^{L R}(d) & =-i\left(g_{1}\right)^{2}(-2 / 9)\left[\overline{d_{L}}(x) \gamma^{\mu} \frac{m_{q^{(1)}}}{\left(p_{q}+p_{B}\right)^{2}-m_{q^{(1)}}^{2}} \gamma^{\nu} d_{R}(x)+H . C .\right] \epsilon_{\mu}^{*}\left(p_{B^{\prime}}\right) \epsilon_{\nu}\left(p_{B}\right) .
\end{aligned}
$$

Since both the K-K bosons and quarks are very massive and the momenta of the external particles are quite small one can employ the non relativistic limit. Thus the helicities of the K-K bosons are space like. Thus to leading order the amplitude corresponding to Eqs (1) and (2) can be written:

$$
\begin{aligned}
\mathcal{M}_{q}^{L}= & -i g_{1}^{2}(1 / 9) \bar{q}(x) \frac{1}{2}\left[\left(-\boldsymbol{\epsilon}^{*^{\prime}} \cdot \boldsymbol{\epsilon}\right) \gamma^{0}-i\left(\boldsymbol{\epsilon}^{*^{\prime}} \times \boldsymbol{\epsilon}\right) \cdot \boldsymbol{\gamma} \gamma_{5}\right] q(x) \times \\
& \left\{\frac{\left(E_{q}+m_{B^{(1)}}\right)}{\left(m_{B^{(1)}}+E_{q}\right)^{2}-m_{q^{(1)}}^{2}}+\frac{\left(E_{q}-m_{B^{(1)}}\right)}{\left(m_{B^{(1)}}-E_{q}\right)^{2}-m_{q^{(1)}}^{2}}\right\}
\end{aligned}
$$

The first term in the square bracket is spin independent and it can lead to coherence. The second term depends on the spin and it can not lead to coherence. It may be important only in the case of odd nuclear targets. The curly bracket in the last equation can be brought into the form:

$$
\frac{E_{q}}{\left(m_{B^{(1)}}\right)^{2}} f_{1}(\Delta), f_{1}(\Delta)=\frac{1+\Delta+\Delta^{2} / 2}{\Delta^{2}(1+\Delta / 2)^{2}}, \Delta=\frac{m_{q^{(1)}}}{m_{B^{(1)}}}-1 .
$$

We see that the amplitude is very sensitive to the parameter $\Delta$ ("resonance effect"). For values of $\Delta$ not very close to zero the event rate is perhaps unobserved since the mass of the $\mathrm{K}-\mathrm{K}$ boson is expected to be large. In the case of the right handed interaction we obtain an analogous expression:

$$
\begin{aligned}
& \mathcal{M}_{u}^{R}=-i g_{1}^{2}(16 / 9) \bar{u}(x) \frac{1}{2}\left[\left(-\boldsymbol{\epsilon}^{*^{\prime}} \cdot \boldsymbol{\epsilon}\right) \gamma^{0}+i\left(\boldsymbol{\epsilon}^{*^{\prime}} \times \boldsymbol{\epsilon}\right) \cdot \boldsymbol{\gamma} \gamma_{5}\right] u(x) \frac{E_{q}}{\left(m_{B^{(1)}}\right)^{2}} f_{1}(\Delta), \\
& \mathcal{M}_{d}^{R}=-i g_{1}^{2}(4 / 9) \bar{d}(x) \frac{1}{2}\left[\left(-\boldsymbol{\epsilon}^{*^{\prime}} \cdot \boldsymbol{\epsilon}\right) \gamma^{0}+i\left(\boldsymbol{\epsilon}^{*^{\prime}} \times \boldsymbol{\epsilon}\right) \cdot \boldsymbol{\gamma} \gamma_{5}\right] d(x) \frac{E_{q}}{\left(m_{B^{(1)}}\right)^{2}} f_{1}(\Delta) .
\end{aligned}
$$

In the case of the R-L interference term there is no $\gamma_{5}$ term. Furthermore the amplitude to leading order is now independent of the energy of the quark. We thus find:

$$
\mathcal{M}_{u}^{R l}=-i g_{1}^{2}(4 / 9) \bar{u}(x)\left[\left(-\boldsymbol{\epsilon}^{*^{\prime}} . \boldsymbol{\epsilon}\right)\right] u(x) \frac{1}{\left(m_{B^{(1)}}\right)} f_{2}(\Delta),
$$




$$
\mathcal{M}_{d}^{R l}=-i g_{1}^{2}(-2 / 9) \bar{d}(x)\left[\left(-\boldsymbol{\epsilon}^{*^{\prime}} \cdot \boldsymbol{\epsilon}\right)\right] d(x) \frac{1}{\left(m_{B^{(1)}}\right)} f_{2}(\Delta)
$$

with

$$
f_{2}(\Delta)=\frac{1+\Delta}{\Delta(1+\Delta / 2)}
$$

The results in this case are less sensitive to $\Delta$.

The next step involves going from the quark to the nucleon level. The only question concerns the quark energy. It seems to us that the best procedure is to replace the quark energy with their constituent mass $\simeq 1 / 3 m_{p}$, as opposed to adopting [1, 2] a procedure related to the current mass encountered in the neutralino case [17, 18], [19]. In the latter case the amplitude at the quark level is proportional to the quark mass (see the intermediate Higgs exchange below). Thus in this case, unlike the neutralino dark matter, the process is dominated by the quarks $u$ and d. So the obtained results do not critically depend on the quark content of the nucleon. We thus find:

$$
\mathcal{M}_{c o h}=i 4 \sqrt{2} G_{F} m_{W} \tan ^{2} \theta_{W}\left(\boldsymbol{\epsilon}^{*^{\prime}} . \boldsymbol{\epsilon}\right) N\left[\left(\frac{11}{18}+\frac{2}{3} \tau_{3}\right) \frac{1}{3} \frac{m_{p} m_{W}}{\left(m_{B^{(1)}}\right)^{2}} f_{1}(\Delta)+\left(\frac{1}{3}+\frac{1}{3} \tau_{3}\right) \frac{m_{W}}{m_{B^{(1)}}} f_{2}(\Delta)\right] N
$$

In Fig. 2 we present the ratio of the amplitude arising from LL and RR contributions alone divided by the total $(\mathrm{LL}+\mathrm{RR}+\mathrm{LR}+\mathrm{RL})$ in the case of the proton. We see that the second term dominates even slightly away from the resonance condition.

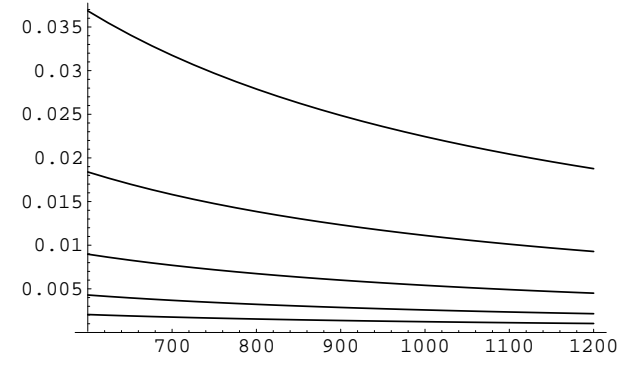

(a)

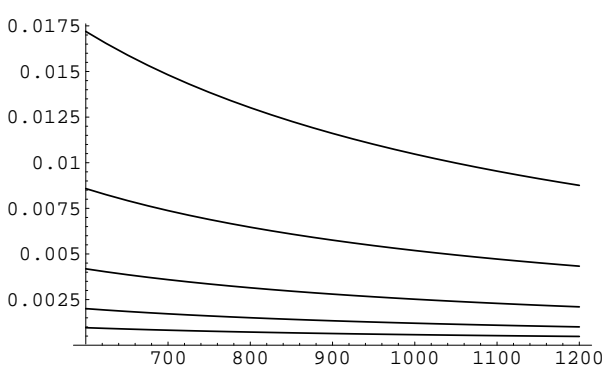

(b)

$$
m_{B^{(1)}} \rightarrow \mathrm{GeV}
$$

$$
m_{B^{(1)}} \rightarrow \mathrm{GeV}
$$

FIG. 2: On the left we show the ratio $(L L+R R) /(L L+R R+L R+R L)$ of the various chirality amplitudes in the case of the proton. On the right we we show the the ratio of the amplitude of the neutron divided by that of the proton. Both are exhibited as functions of $m_{B^{(1)}}$ in $\mathrm{GeV}$ for the values of $\Delta=0.05,0.10,0.20$ and 0.40 (from top to bottom). We see that due to the LR+RL contribution the amplitude associated with the proton is dominant.

In the case of the spin contribution we find at the quark level that:

$$
\mathcal{M}_{\text {spin }}=-i 4 \sqrt{2} G_{F} m_{W} \tan ^{2} \theta_{W} \frac{1}{3} \frac{m_{p} m_{W}}{\left(m_{B^{(1)}}\right)^{2}} f_{1}(\Delta) \bar{q} i\left(\boldsymbol{\epsilon}^{*^{\prime}} \times \boldsymbol{\epsilon}\right) \cdot\left[\frac{17}{18} \bar{u} \gamma \gamma_{5} u+\frac{5}{18} \bar{d} \gamma \gamma_{5} d+\frac{5}{18} \bar{s} \boldsymbol{\gamma} \gamma_{5} s\right]
$$

In going to the nucleon level we get the isoscalar part [19]

$$
g_{0}=\frac{17}{18} \Delta u+\frac{5}{18} \Delta d+\frac{5}{18} \Delta s
$$

while the isovector part is:

$$
g_{1}=\frac{17}{18} \Delta u-\frac{5}{18} \Delta d
$$


The quantities $\Delta_{q}$ are given by [1, 2, 19]

$$
\Delta u=0.78 \pm 0.02, \Delta d=-0.48 \pm 0.02, \Delta s=-0.15 \pm 0.02 .
$$

We thus find,

$$
g_{0}=0.26, g_{1}=0.41 \text {. }
$$

In the proton neutron representation we obtain:

$$
a_{p}=0.67, a_{n}=-0.15 .
$$

The picture is different for the neutralino case in which [20],

$$
g_{0}=\Delta u+\Delta d+\Delta s=0.15, g_{1}=\Delta u-\Delta d=1.26, a_{p}=1.41, a_{n}=-1.11 .
$$

Thus at the nucleon level we get

$$
\mathcal{M}_{\text {spin }}=-i 4 \sqrt{2} G_{F} m_{W} \tan ^{2} \theta_{W} \frac{1}{3} \frac{m_{p} m_{W}}{\left(m_{B^{(1)}}\right)^{2}} f_{1}(\Delta) \bar{q} i\left(\boldsymbol{\epsilon}^{\boldsymbol{*}^{\prime}} \times \boldsymbol{\epsilon}\right) .\left[N \boldsymbol{\sigma}\left(g_{0}+g_{1} \tau_{3}\right) N\right] .
$$

\section{Intermediate Higgs Scalars}

The corresponding Feynman diagram is shown in Fig. 3 The vertex involving the interaction of

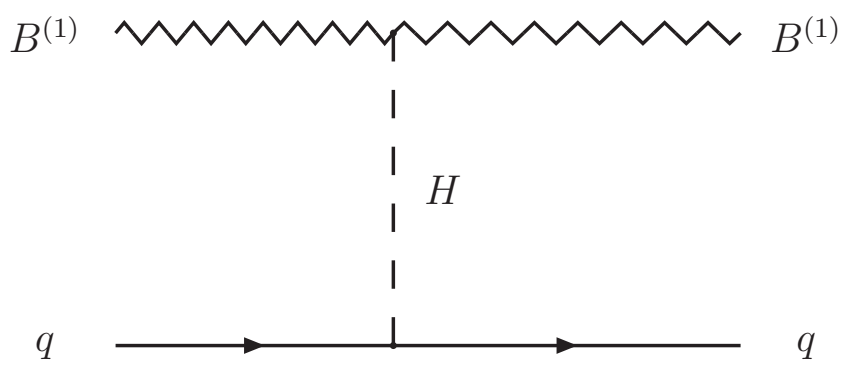

FIG. 3: The interaction of K-K gauge boson $B^{(1)}$ with quarks at tree level mediated by Higgs scalars

the Higgs particle with the $\mathrm{K}-\mathrm{K}$ boson is given by

$$
\mathcal{L}_{B B h}=g_{1}^{2} \frac{1}{4} \epsilon_{\nu}^{*}\left(p_{B}^{\prime}\right) \epsilon_{\nu}\left(p_{B}\right) H H \Rightarrow g_{1}^{2} \frac{1}{4} \epsilon_{\nu}^{*}\left(p_{B}^{\prime}\right) \epsilon_{\nu}\left(p_{B}\right) h \prec H \succ .
$$

The coupling of the Higgs scalar to the quark is given by:

$$
\mathcal{L}_{q q h}=\frac{m_{q}}{\prec H \succ} h .
$$

We thus obtain

$$
\mathcal{M}_{q}(h)=-i g_{1}^{2} \frac{1}{4}\left[-\epsilon^{*^{\prime}} \cdot \boldsymbol{\epsilon} \bar{q}(x) \frac{m_{q}}{m_{h}^{2}} q(x)\right] .
$$

In going from the quark to the nucleon level we follow a procedure analogous to that of the of the neutralino [17, 18, 19], i.e.

$$
\prec N\left|m_{q} q \bar{q}\right| N \succ \Rightarrow f_{q} m_{p},
$$

we thus get

$$
\mathcal{M}_{N}(h)=-i 4 \sqrt{2} G_{F} m_{W}^{2} \tan ^{2} \theta_{W}\left[\frac{1}{4} \frac{m_{p}}{m_{h}^{2}}\left(-\boldsymbol{\epsilon}^{*^{\prime}} . \boldsymbol{\epsilon}\right) \prec N \mid N \succ \sum_{q} f_{q}\right] .
$$

In this case the proton and the neutron cross sections are about equal. 


\section{K-K NEUTRINOS AS DARK MATTER CANDIDATES}

The other possibility is the dark matter candidate to be a heavy $K-K$ neutrino. We distinguish the following cases

\section{Process mediated by Z-exchange}

The Feynman diagram associated with this process is shown in Fig. 4. The $q q Z$ vertex is given

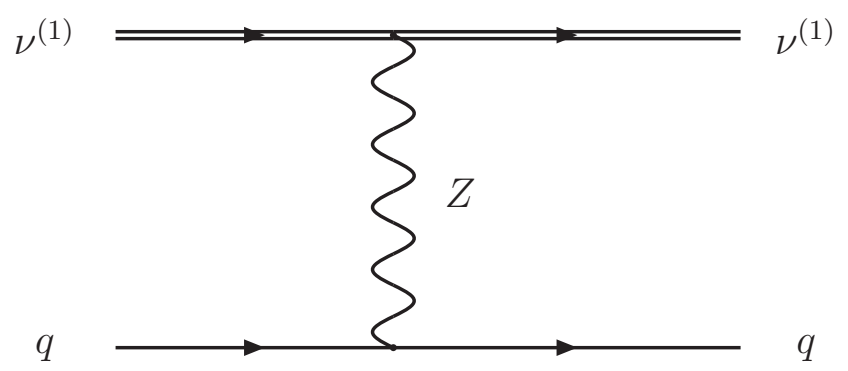

FIG. 4: The interaction of K-K neutrino $\nu^{(1)}$ with quarks at tree level mediated by Z-exchange

by,

$$
\begin{gathered}
f_{\lambda}=-\frac{1}{2} \frac{g}{2 \cos \theta_{W}} J_{\lambda}(q q Z), \\
J_{\lambda}(q q Z)=\bar{q} \gamma_{\lambda}\left[-2 \sin ^{2} \theta_{W}\left(\frac{1}{3}+\tau_{3}\right)+\left(1-\gamma_{5}\right) \tau_{3}\right] q, \\
J_{\lambda}(N N Z)=\bar{N} \gamma_{\lambda}\left[-2 \sin ^{2} \theta_{W}\left(1+\tau_{3}\right)+\left(1-g_{A} \gamma_{5}\right) \tau_{3}\right] N .
\end{gathered}
$$

Thus in the case for the proton we encounter the combination,

$$
\left(-g_{A} \gamma_{5}+1-4 \sin ^{2} \theta_{W}\right) \simeq-g_{A} \gamma_{5}
$$

while in the case of the neutron:

$$
-1+g_{A} \gamma_{5}
$$

which exhibit the well known fact the in the case of the neutral current interaction we can have coherence over the neutrons, but no coherence over the protons. Thus,

$$
J_{\lambda}(N N Z) \simeq \bar{N} \gamma_{\lambda}\left[-g_{A} \gamma_{5} \tau_{3}-\frac{1}{2}\left(1-\tau_{3}\right] N\right.
$$

In the case of the neutrino vertex we write:

$$
f_{\lambda}\left(\nu^{(1)}\right)=-\frac{1}{2} \frac{g}{2 \cos \theta_{W}} J_{\lambda}\left(\nu^{(1)}\right) .
$$

Regarding the neutrino current we now have two possibilities:

- the K-K neutrino is a Majorana particle. In this case the neutrino current is:

$$
J_{\lambda}\left(\nu^{(1)}\right)=\bar{\nu}^{(1)} \gamma_{\lambda} \gamma_{5} \nu^{(1)}
$$

The Majorana neutrino has no electromagnetic properties (no neutral vector current interaction). 
- The K-K neutrino is a Dirac particle. In the presence of left handed interaction only we have:

$$
J_{\lambda}\left(\nu^{(1)}\right)=\bar{\nu}^{(1)} \gamma_{\lambda}\left(1-\gamma_{5}\right) \nu^{(1)}
$$

The amplitude associated with the diagram of Fig. 4 becomes:

$$
\mathcal{M}_{\nu^{(1)}}=\frac{1}{4} \frac{g^{2}}{4 \cos ^{2} \theta_{W}} \frac{1}{-m_{Z}^{2}} J^{\lambda}\left(\nu^{(1)}\right) J_{\lambda}(N N Z)=-\frac{1}{2 \sqrt{2}} G_{F} J^{\lambda}\left(\nu^{(1)}\right) J_{\lambda}(N N Z) .
$$

In other words in the case of the Majorana neutrino we get:

$$
\mathcal{M}_{\nu^{(1)}}=\frac{1}{2 \sqrt{2}} G_{F} \bar{N} \gamma_{\lambda} g_{A} \gamma_{5} \tau_{3} N \bar{\nu}^{(1)} \gamma_{\lambda} \gamma_{5} \nu^{(1)}
$$

while in the case of a Dirac neutrino the coherent contribution dominates, i.e.:

$$
\mathcal{M}_{\nu^{(1)}}=\frac{1}{2 \sqrt{2}} G_{F} \bar{N} \gamma_{\lambda} \frac{1-\tau_{3}}{2} N \bar{\nu}^{(1)} \gamma_{\lambda}\left(1-\gamma_{5}\right) \nu^{(1)}
$$

\section{Process mediated by right handed currents via $Z^{\prime}$-boson exchange}

The process is similar to that exhibited by Fig. 4, except that instead of Z we encounter $Z^{\prime}$, which is much heavier. We will assume that the couplings of the $Z^{\prime}$ are similar to those of $Z$. Then the above results apply except that now the amplitudes are retarded by the multiplicative factor $\kappa=m_{Z}^{2} / m_{Z^{\prime}}^{2}$

\section{Process mediated by Higgs exchange}

In this case in Fig. 4, $\mathrm{Z}$ is replaced by the Higgs particle. In this case the amplitude at the quark level becomes:

$$
\mathcal{M}_{\nu^{(1)}}(h)=-2 \sqrt{2} G_{F} \frac{m_{q} m_{\nu^{(1)}}}{m_{h}^{2}} \bar{\nu}^{(1)} \nu^{(1)} \bar{q} q .
$$

Proceeding as above we find that the amplitude at the nucleon level is:

$$
\mathcal{M}_{\nu^{(1)}}(h)=-2 \sqrt{2} G_{F} \frac{m_{p} m_{\nu^{(1)}}}{m_{h}^{2}} \bar{\nu}^{(1)} \nu^{(1)} \prec N \mid N \succ \sum_{q} f_{q} .
$$

\section{NUCLEON CROSS SECTIONS}

In evaluating the nucleon cross section one proceeds as in the case of the neutralino. The momentum transfer to the nucleon is $q=2 \mu_{r} v \xi$, where $\mu_{r}=$ reduced mass $\simeq m_{p}, v$ is the dark matter candidate velocity and $\xi$ is the cosine of the angle between the initial dark matter particle and the outgoing nucleus.

\section{The K-K boson case}

To obtain the nucleon cross section, one must sum over the final spin and boson polarizations and average over the initial ones. One finds,

$$
\sigma_{N}(c o h)=\frac{1}{4 \pi} \frac{m_{p}^{2}}{\left(m_{B^{(1)}}\right)^{2}} \frac{1}{2} \frac{1}{3} \sum_{p o l, m_{s}}\left|\mathcal{M}_{c o h}+\mathcal{M}_{N}(h)\right|^{2},
$$




$$
\sigma_{N}(\operatorname{spin})=\frac{1}{4 \pi} \frac{m_{p}^{2}}{\left(m_{B^{(1)}}\right)^{2}} \frac{1}{2} \frac{1}{3} \sum_{\text {pol }, m_{s}}\left|\mathcal{M}_{\text {spin }}\right|^{2}
$$

where,

$$
\frac{1}{2} \frac{1}{3} \sum_{p o l, m_{s}}=1, \frac{1}{2} \frac{1}{3} \sum_{p o l, m_{s}}=6,
$$

for the spin independent and spin dependent parts respectively.

Unlike the neutralino case, where one has to live with an allowed SUSY parameter space involving 4 parameters, see e.g. Ellis et al [21], Bottino et al, and Arnowitt et al [22], the situation here is much simpler. One encounters only three mass parameters, $\Delta, m_{B^{(1)}}, m_{h}$, in the case of the coherent process and only the first two parameters in the case of the spin cross section. Admittedly, however, the cross section depends on large powers of these masses and, therefore, the predictions of the event rates are not very accurate. The isoscalar spin cross section and the Higgs contribution of the coherent process depend on the structure of the nucleon. The uncertainties encountered here are no worse than those in the neutralino cross section. In the evaluation of the parameters $f_{q}$ one encounters both theoretical and experimental errors. Thus the nucleon cross section associated with the Higgs mechanism can vary within an order of magnitude [19]. In the present calculation we will adopt an optimistic approach and employ:

$$
f_{d}=0.041, f_{u}=0.028, f_{s}=0.400, f_{c}=0.051, f_{b}=0.055, f_{t}=0.095 .
$$

The thus obtained results for the coherent process are shown in Fig. 5. The independent variable in our plots is the mass of the dark matter candidate, since this has become standard in analyzing the experimental searches. The cross sections associated with intermediate Higgs scalars only are plotted in Fig. 口 below. In the case of the spin cross section we obtain the 3-dimensional plots shown in Fig. 6. Since the cross sections, especially the spin cross sections, are sensitive functions of their arguments we will present the above results a a series of one dimensional plots. This is done in Fig. 7 for the coherent mode and in Fig. 8 for that of the spin.

\section{The K-K neutrino case}

In this case the expression for the cross section is quite simple. We will consider each case separately.

Intermediate $Z$ boson.

In this case we have two possibilities:

- Majorana neutrino. Now only the axial current contributes. The proton and the neutron cross sections are equal and given by:

$$
\sigma_{N}(\operatorname{spin})=\frac{1}{\pi} \frac{G_{F}^{2}}{8} m_{p}^{2} 3 g_{A}^{2}=8.0 \times 10^{-3} p b .
$$

- Dirac neutrino. In this case we have again a contribution due to the axial current, but the resulting nucleon cross section is twice as large compared to the previous case, i.e.:

$$
\sigma_{N}(\operatorname{spin})=\frac{1}{\pi} \frac{G_{F}^{2}}{8} m_{p}^{2} 32 g_{A}^{2}=1.6 \times 10^{-2} p b .
$$

In the case of the neutron we have, however, an additional spin independent contribution given by:

$$
\sigma_{n}(c o h)=\frac{1}{\pi} \frac{G_{F}^{2}}{8} m_{p}^{2} 2=3.5 \times 10^{-3} p b .
$$




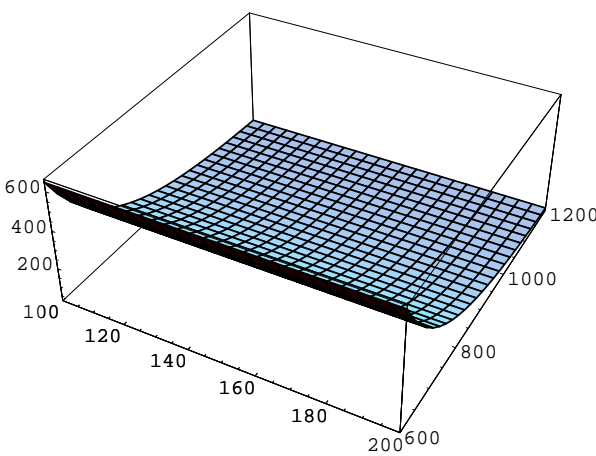

(a)

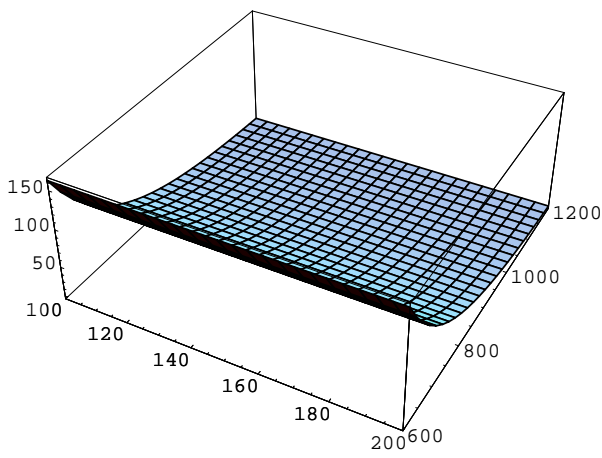

(c)

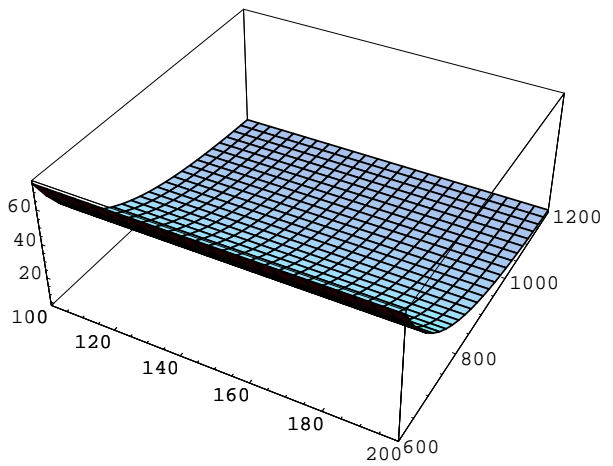

(e)

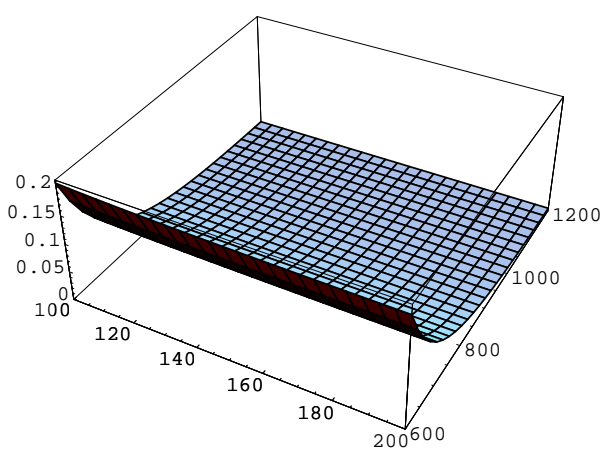

(b)

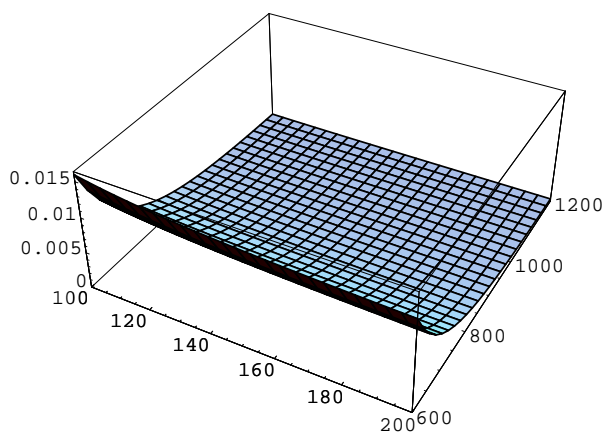

(d)

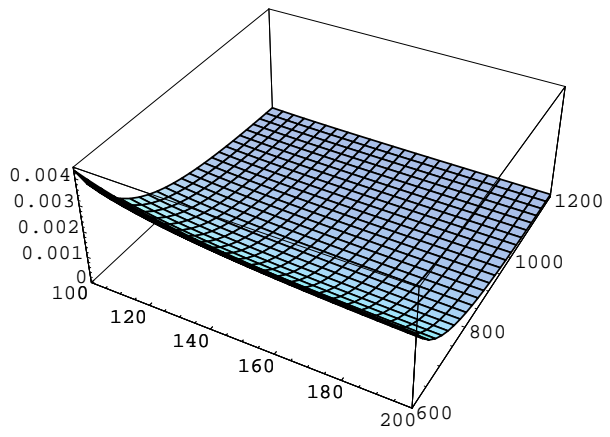

(f)

FIG. 5: The coherent proton cross section on the left and that for the neutron on the right in units of $10^{-6} \mathrm{pb}$, as a function of the gauge boson mass in the range of $600-1200 \mathrm{GeV}$ and the Higgs mass in the range of $100-200 \mathrm{GeV}$. From top to bottom $\Delta=0.05,0.10$ and 0.15 respectively

It is quite straightforward to compute the nuclear cross sections:

$$
\sigma_{\text {nuclear }}(\operatorname{spin})=\frac{\mu_{r}^{2}}{m_{p}^{2}} \sigma_{N}(\operatorname{spin}) \zeta_{\text {spin }} F_{11}(q)
$$

Here $F_{11}$ is the spin response function [19, 23, 24], which depends on the energy $Q$ transfered to the nucleus, $u=m_{A} Q b^{2}$, with $b$ the nuclear harmonic oscillator size parameter, and $\zeta_{\text {spin }}$ is the nuclear 


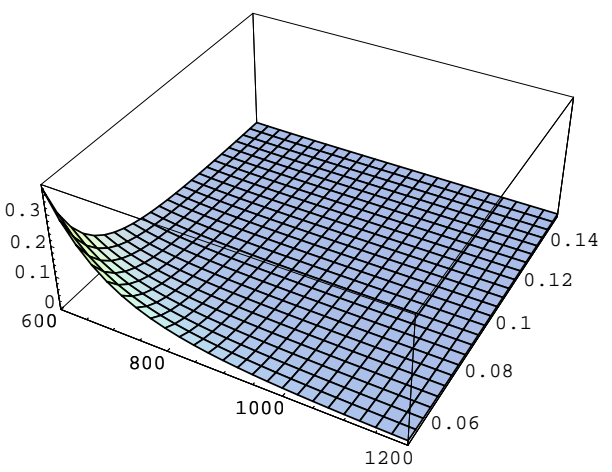

(a)

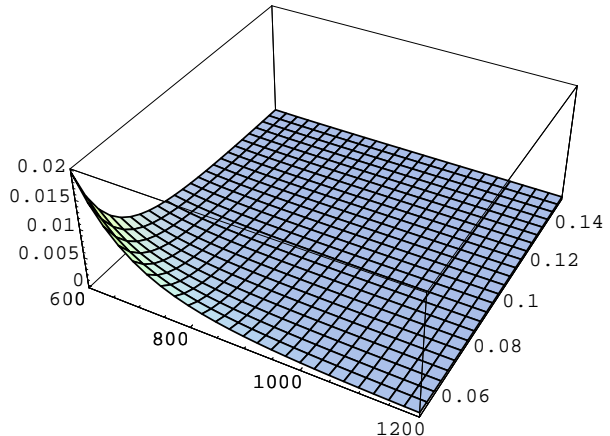

(b)

FIG. 6: The spin proton cross section on the left and that for the neutron on the right in units of $10^{-6} \mathrm{pb}$, as a function of the gauge boson mass in the range of $600-1200 \mathrm{GeV}$ and $\Delta$ in the range $0.05-0.15$.

static spin ME given by:

$$
\zeta_{\text {spin }}=\frac{1}{3}\left[\Omega_{p}-\Omega_{n} \sqrt{\frac{\sigma_{n}}{\sigma_{p}}}\right]^{2}=\frac{1}{3}\left[\Omega_{p}-\Omega_{n}\right]^{2}
$$

$\left(\sigma_{p}=\sigma_{n}=\sigma_{N}\right)$. Here $\Omega_{p}$ and $\Omega_{n}$ are the nuclear spin ME associated with the proton and neutron component respectively.

The coherent cross section becomes

$$
\sigma_{\text {nuclear }}(\operatorname{coh})=\frac{\mu_{r}^{2}}{m_{p}^{2}} \sigma_{n}(\operatorname{coh}) N^{2}[F(q)]^{2},
$$

where $N$ is the neutron number and $F(q)$ the nuclear form factor.

\section{The right handed interaction.}

In this case case the nucleon cross section is retarded compared to the previous case. The obtained results are shown in Fig. 9

The Intermediate Higgs scalar.

Naively one expects this process to be suppressed due to the small mass of the $\mathrm{u}$ and $\mathrm{d}$ quarks [25], present in the nucleon. This is true in the naive quark model for the nucleon. We have seen above and we know from the neutralino case that the heavy quarks contribute and in fact dominate. One finds:

$$
\sigma_{N}(\operatorname{coh})=\frac{8}{\pi}\left(G_{F} m_{p}^{2}\right)^{2} \frac{m_{p}^{2}\left(m_{\nu^{(1)}}\right)^{2}}{m_{h}^{4}} m_{p}^{-2}\left(\sum_{q} f_{q}\right)^{2}=1.1 \times 10^{-1} p b \frac{m_{p}^{2}\left(m_{\nu^{(1)}}\right)^{2}}{m_{h}^{4}}\left(\sum_{q} f_{q}\right)^{2} .
$$

The value $\sum_{q} f_{q}=0.67$ is acceptable. Using this value we obtain the results shown in Fig. 10, We see that this mechanism excludes a heavy neutrino as a WIMP candidate, unless the Higgs mass is much larger. In the Standard Model this is possible and $m_{h}$ can be treated as a parameter to be extracted from the data. In SUSY models, however, the lightest neutrino is expected to be quite light, $m_{h} \simeq 120 \mathrm{GeV}$. 


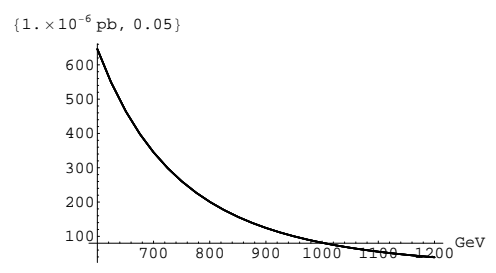

(a)

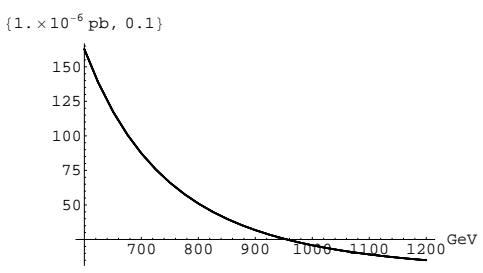

(c)

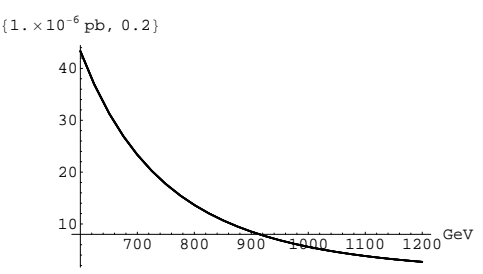

(e)

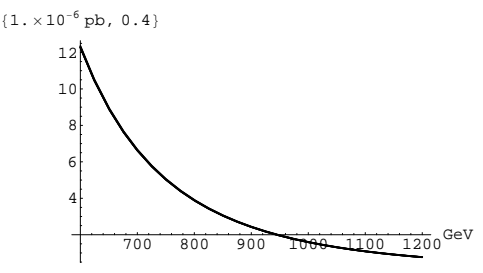

(g)

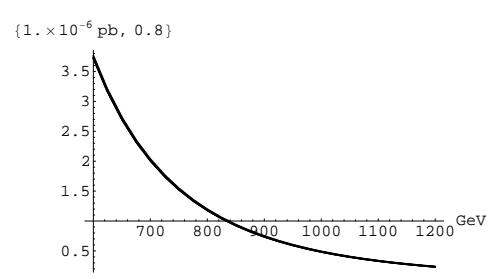

(i)

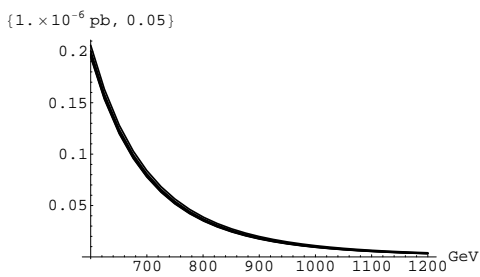

(b)

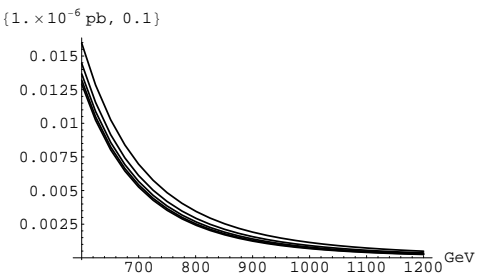

(d)

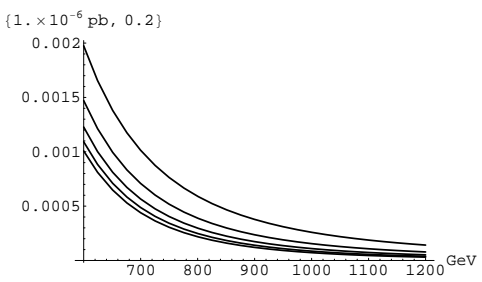

(f)

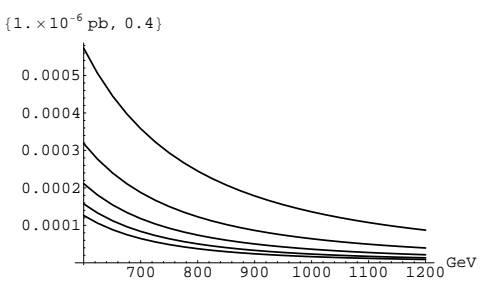

(h)

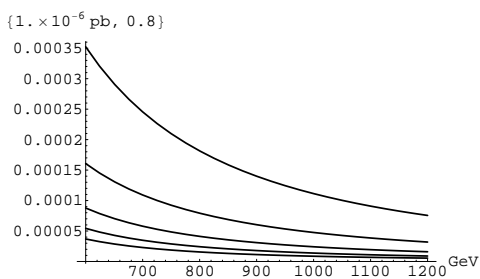

(j)

FIG. 7: The spin independent proton cross section on the left and that for the neutron on the right in units of $10^{-6} \mathrm{pb}$, as a function of the gauge boson mass in the range of $600-1200 \mathrm{GeV}$. ¿From top to bottom $\Delta=0.05,0.10,0.20,0.40$ and 0.80 . On each plot we show results for $m_{h}=100,125,150,175$ and $200 \mathrm{GeV}$ with mass increasing downwards. Note that in the case of the proton, due to the RL+LR dominance, the effect of Higgs contribution is not visible. 


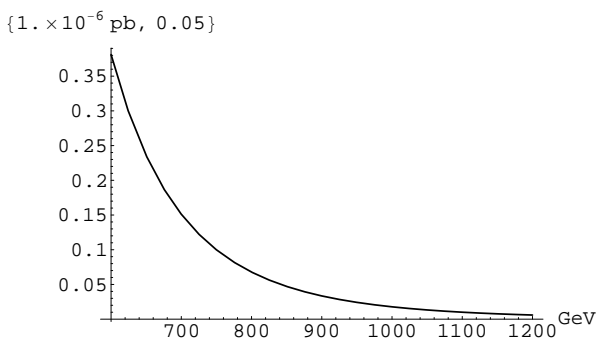

(a)

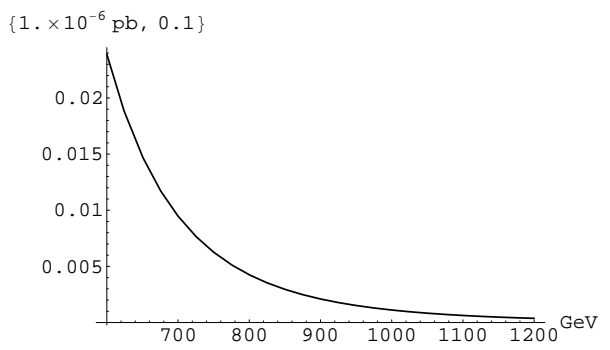

(c)

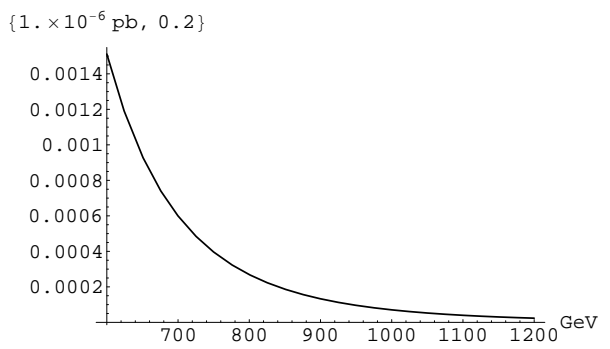

(e)

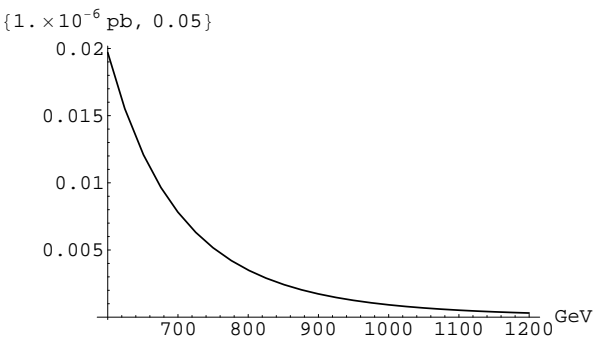

(b)

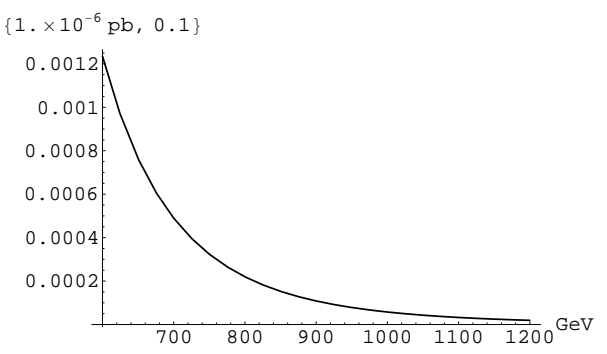

(d)

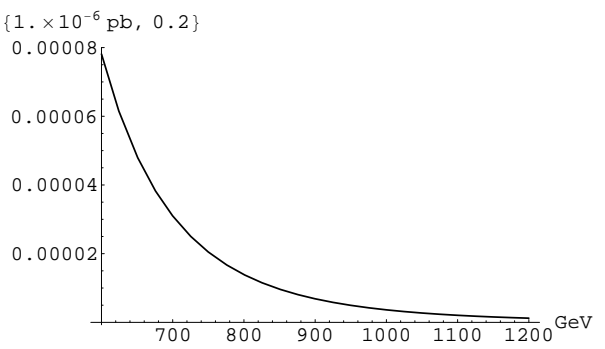

(f)

FIG. 8: The spin proton cross section on the left and that for the neutron on the right in units of $10^{-6} \mathrm{pb}$, as a function of the gauge boson mass in the range of $600-1200 \mathrm{GeV}$. From top to bottom $\Delta=0.05,0.10$ and 0.20 respectively.

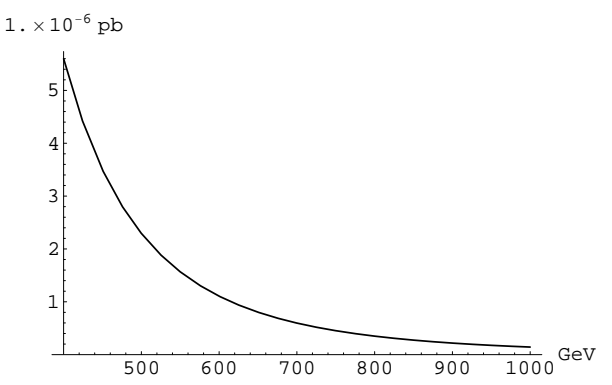

(a)

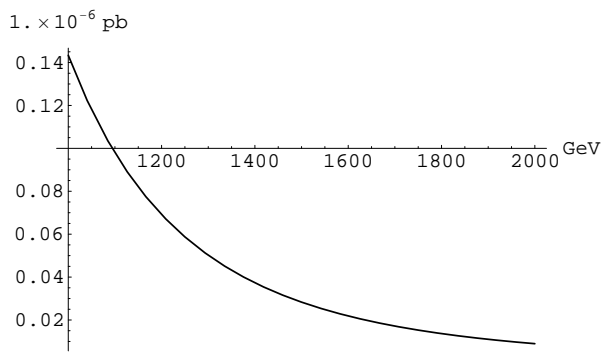

(b)

FIG. 9: The coherent nucleon cross section in the case of right handed neutrino interaction for a Dirac $\nu^{(1)}$ as a function of the gauge boson mass responsible for this interaction. 


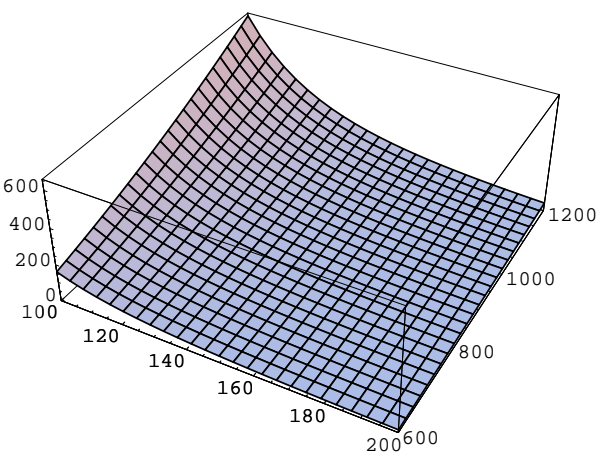

(a)

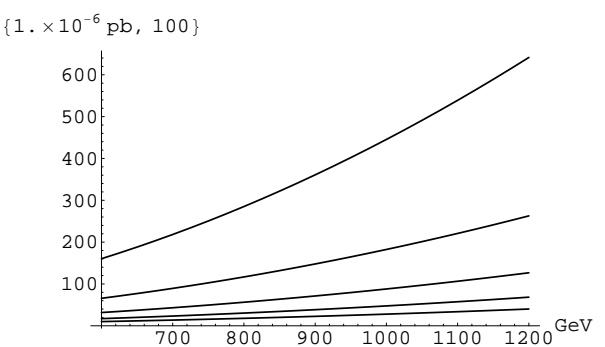

(b)

FIG. 10: On the left we show the coherent nucleon cross section as a function of $m_{\nu^{(1)}}$ and $m_{h}$ in GeV. On the right we show the same thing as a function of the mass of $\nu^{(1)}$ for the indicated Higgs mass (from top to bottom 100, 125, 150, 175 and $200 \mathrm{GeV}$ ). We see that this mechanism excludes a heavy neutrino as a WIMP candidate, unless the Higgs mass is quite large.

\section{OTHER NON SUSY MODELS}

There exist extensions of the Standard Model not motivated by symmetry, which may have a particle content similar to that of K-K theories discussed above. Such models, however, have a much lower predictive power than the K-K scenarios discussed above. To see this we examine the following cases:

- Models which introduce extra Higgs particles and impose a discrete symmetry which leads to a "parity" a la R-parity or K-K parity [26].

- Extensions of the Standard Model, which do not require the ad hoc introduction of a parity, but introduce high weak isospin multiplets [27] with $\mathrm{Y}=0$. So the WIMP-nucleus interaction via Z-exchange at tree level is absent and the dominant contribution to the WIMP-nucleus scattering occurs at the one loop level.

We will consider here the first of the above possibilities [26]. All particles of the Standard Model have parity +1 , while the new exotic particles have parity -1 . The Standard Model particles of interest to us here, leptons and Higgs, are:

$$
\begin{aligned}
& \left(\nu_{i}, l_{i}\right) \sim(2,-1 / 2), \quad l_{i}^{c} \sim(1,1), \quad N_{i} \sim(1,0), \\
& \left(\phi^{+}, \phi^{0}\right) \sim(2,1 / 2), \quad\left(\eta^{+}, \eta^{0}\right) \sim(2,1 / 2) .
\end{aligned}
$$

in the usual notation $S U(2)_{L} \times U(1)_{Y}$ quantum numbers. Consider now the following minimal extension of the SM with symmetry $S U(2)_{L} \times U(1)_{Y} \times Z_{2}$ and particle content:

$$
\begin{aligned}
& \left(\nu_{i}, l_{i}\right) \sim(2,-1 / 2 ;+), \quad l_{i}^{c} \sim(1,1 ;+), \quad N_{i} \sim(1,0 ;-), \\
& \left(\phi^{+}, \phi^{0}\right) \sim(2,1 / 2 ;+), \quad\left(\eta^{+}, \eta^{0}\right) \sim(2,1 / 2 ;-) .
\end{aligned}
$$

Note that the particles $N_{i}$ and the scalar doublet $\left(\eta^{+}, \eta^{0}\right)$ are odd under $Z_{2}$. This makes the lightest exotic particle a viable dark matter candidate. In other words in this economic scenario one introduces:

- A new doublet of Higgs scalars $\eta$, which have the same quantum numbers with the ordinary Higgs, but parity -1 . These are expected to be quite massive, but they do not develop a vacuum expectation value.

- Assign parity -1 to the usual isosinglet right handed neutrinos. 
In this scenario the see-saw mechanism for neutrino mass generation is not operative. One can not have a Dirac mass term $\bar{\nu}_{i L} \phi^{0} N_{j R} \rightarrow \bar{\nu}_{i L} \prec \phi^{0} \succ N_{j R}$ since the "parity" forbids it. One can have Majorana mass terms at the one loop level as shown in Fig. 11, involving two $\eta$ scalars. The two $\eta$ scalars couple with the ordinary Higgs scalars with a quartic coupling $\lambda$. The net result is that the isosinglet neutrino can be much lighter than that of the the standard see-saw mechanism. We now

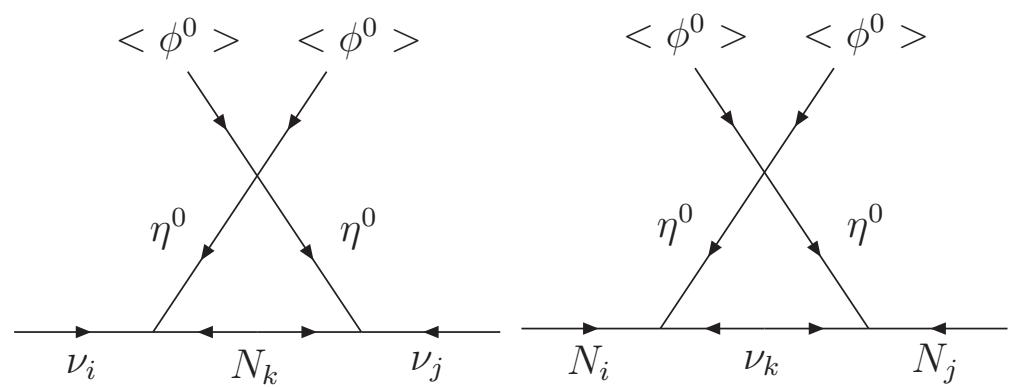

FIG. 11: The 1-loop diagram responsible for the Majorana neutrino masses. Note that, since the isosinglet neutrino has negative $Z_{2}$ parity, there is no Dirac mass. An adjustable quartic coupling $\lambda$ is understood.

have two possibilities

- The lightest of the heavy neutrinos is the dark matter candidate.

In this case the obtained results are the same as those discussed in the previous sections in connection with the right handed interaction (see Fig. 9).

- The neutral component of the exotic Higgs scalars $\eta$ is the dark matter candidate. In this case, since we have not introduced exotic quarks, the interaction of the dark matter candidate with the quarks is achieved only via the ordinary Higgs (see Fig. 12, The corresponding effective

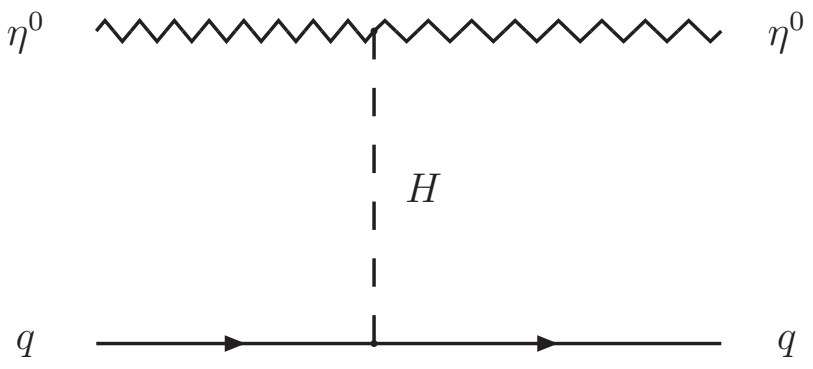

FIG. 12: The The mechanism leading do direct dark matter detection for a scalar WIMP $\eta^{0}$, which is stable to the fact that it has $Z_{2}$ parity -1 . This is similar with Fig 3 except that an effective coupling $\lambda_{e f f}=\tilde{\lambda}\left(g_{1}^{2} / 4\right)$, with $\tilde{\lambda}$ treated phenomenologically, is understood.

$\eta \eta h$ coupling is now parameterized as $\lambda_{\text {eff }}=\tilde{\lambda}\left(g_{1}^{2} / 4\right)(\tilde{\lambda}=1$ corresponds to the K-K case). Applying the formalism of the previous section we obtained results like those shown in Fig. 口

As in the case of K-K theories, only in the next generation of experiments such WIMP's can be detected.

Before concluding this section we should mention another interesting extension of the Standard Model in the direction of technicolour [28]. In this case the WIMP is the neutral LTP (lightest neutral technibaryon). This is scalar particle, which couples to the quarks via derivative coupling through Z-exchange. This model, however, in its present form, leads to too large nucleon cross sections and is excluded by the data. 


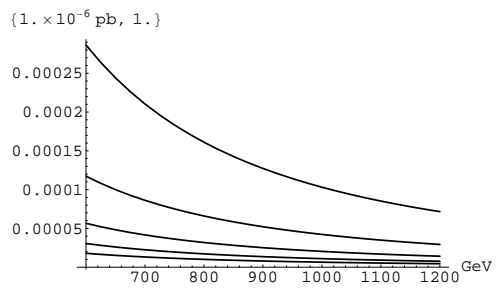

(a)

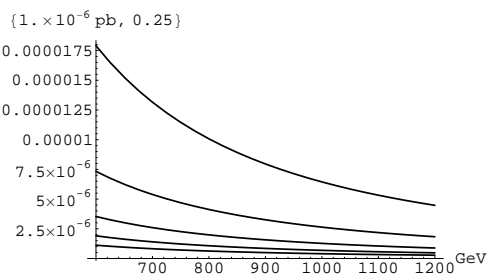

(c)

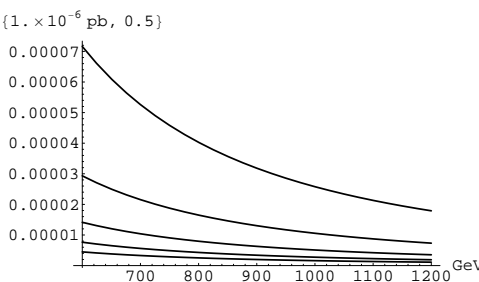

(b)

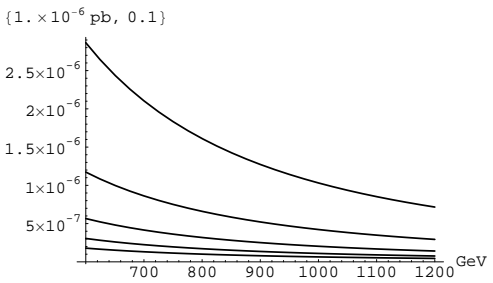

(d)

FIG. 13: The nucleon cross section corresponding to the $\eta$ scalar being the dark matter candidate, as a function of the $\eta$ mass. On each graph we present results for $m_{h}=100,125,150,175$ and $200 \mathrm{GeV}$ (the mass increases downwards). ¿From top to bottom and left to right $\tilde{\lambda}=1,0.5,0.25$ and 0.1 respectively. Note that the special case $\tilde{\lambda}=1$ it coincides with the Higgs contribution discussed above in connection with the $\mathrm{K}-\mathrm{K}$ theories.

\section{EVENT RATES}

The event rate for the coherent WIMP-nucleus elastic scattering is given by [19, 29]:

$$
R=\frac{\rho(0)}{m_{\chi^{0}}} \frac{m}{m_{p}} \sqrt{\left\langle v^{2}\right\rangle}\left[f_{\text {coh }}\left(A, \mu_{r}(A)\right) \sigma_{p, \chi^{0}}^{S}+f_{\text {spin }}\left(A, \mu_{r}(A)\right) \sigma_{p, \chi^{0}}^{\text {spin }} \zeta_{\text {spin }}\right],
$$

with

$$
f_{\text {coh }}\left(A, Z, \mu_{r}(A)\right)=\frac{100 \mathrm{GeV}}{m_{\chi^{0}}}\left[\frac{\mu_{r}(A)}{\mu_{r}(p)}\right]^{2} \frac{g_{c o h}^{2}(A, Z)}{A} t_{c o h}\left(1+h_{c o h} \cos \alpha\right),
$$

and $g_{c o h}(A, Z)=A, A-Z, Z$ for total, neutron and proton coherence respectively.

$$
f_{\text {spin }}\left(A, \mu_{r}(A)\right)=\frac{100 \mathrm{GeV}}{m_{\chi^{0}}}\left[\frac{\mu_{r}(A)}{\mu_{r}(p)}\right]^{2} \frac{t_{\text {spin }}}{A}\left(1+h_{\text {spin }} \cos \alpha\right),
$$

with $\sigma_{p, \chi^{0}}^{S}$ and $\sigma_{p, \chi^{0}}^{\text {spin }}$ the scalar and spin proton cross sections $\zeta_{\text {spin }}$ the nuclear spin ME.

In this work we will ignore the motion of the earth, i.e. $h_{c o h}=h_{\text {spin }}=0$ no modulation.

\section{The coherent contribution due to the scalar interaction.}

The number of events in time $t$ due to the scalar interaction, which leads to coherence, is:

$$
R \simeq 1.6010^{-3} \frac{t}{1 \mathrm{y}} \frac{\rho(0)}{0.3 G e V c m^{-3}} \frac{m}{1 \mathrm{Kg}} \frac{\sqrt{\left\langle v^{2}\right\rangle}}{280 \mathrm{kms}^{-1}} \frac{\sigma_{p, \chi^{0}}^{S}}{10^{-6} \mathrm{pb}} f_{\text {coh }}\left(A, Z, \mu_{r}(A)\right) .
$$

The parameter $t$ depends on the structure of the nucleus, the WIMP velocity distribution, the WIMP mass and the energy cutoff imposed by the detector. In the case of ${ }^{127} \mathrm{I}$ this parameter is shown in Fig. 14. The nucleon cross section depends on the particle model. We will consider the following cases: 


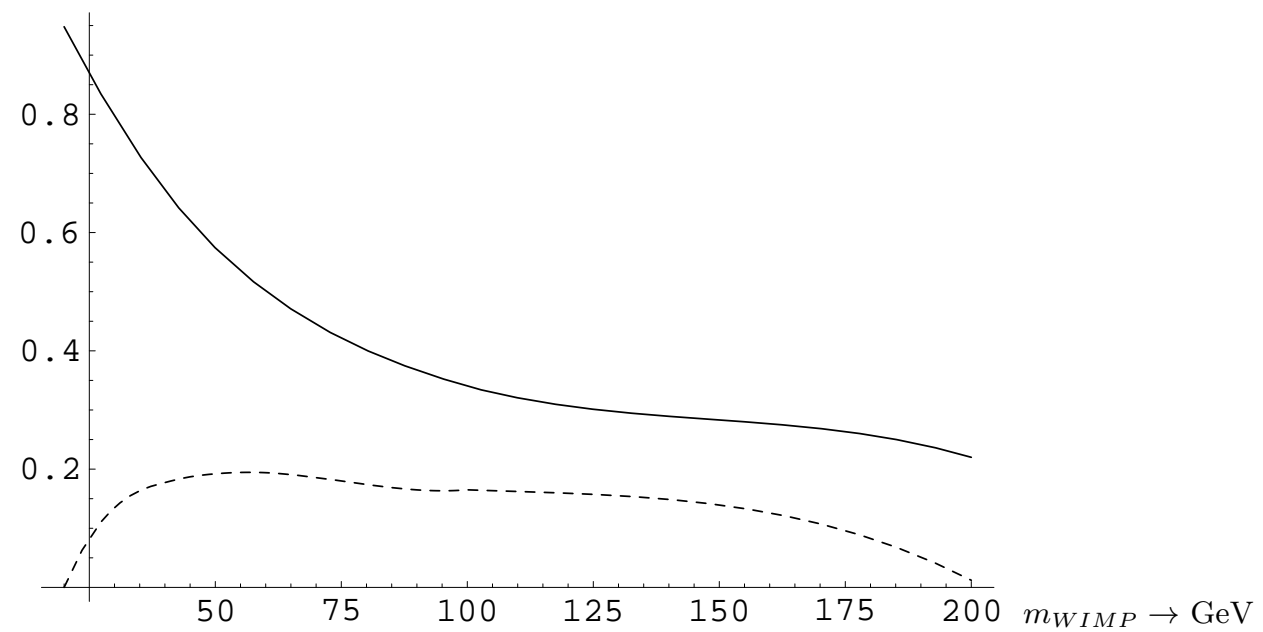

FIG. 14: The parameter $t$ in the case of ${ }^{127} \mathrm{I}$ as a function of the WIMP mass in GeV for zero threshold (continuous curve) and a threshold of $10 \mathrm{keV}$ (dotted curve). For higher WIMP masses $t$ remains approximately constant.

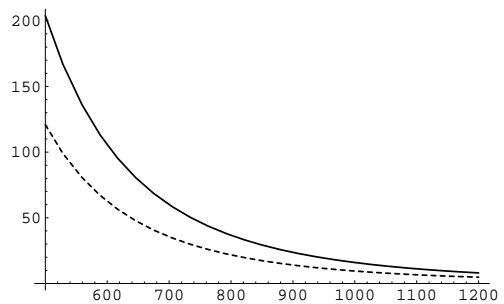

(a)

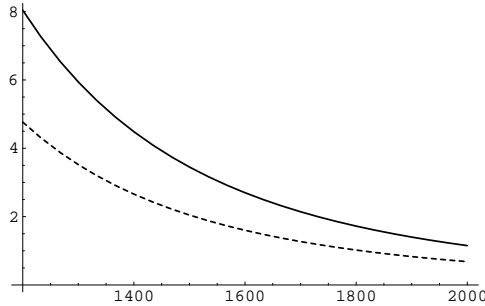

(b) $\quad m_{W I M P} \rightarrow \mathrm{GeV}$

FIG. 15: The coherent event rate $R$ per year per $\mathrm{Kg}$ of target in the case of ${ }^{127} \mathrm{I}$, in the case of the K-K gauge boson as WIMP, plotted a as function of the WIMP mass in GeV for zero threshold (continuous curve) and a threshold of $10 \mathrm{keV}$ (dotted curve). Both figures show the same quantity except the WIMP masses range is different.

- The WIMP is a K-K boson

In this case we will consider the viable possibility $\Delta=0.8$ (see Fig. 7). Then one obtains the event rates shown in Fig. 15. We see that, even further from the degeneracy and quite heavy WIMPs, $m_{\chi} \simeq 1 \mathrm{TeV}$, the event rates are detectable.

- The WIMP is a K-K Majorana neutrino.

The Dirac K-K neutrino case is excluded, since, then, the Z-induced neutron coherent contribution would be too large. In the case of Majorana neutrinos one can have coherence due to the amplitude obtained via the Higgs exchange. The obtained results are shown in Fig. 16. From this figure we see that the lighter Higgs mass is allowed by the data only for relatively light WIMPs. The heavier Higgs mass, however, is allowed for all WIMPs. Clearly such heavy Higgs cannot occur in SUSY theories, since then, $m_{h} \leq 120 \mathrm{GeV}$.

\section{The spin interaction.}

In this case, the event in time $t$ rate can be cast into the form:

$$
R \simeq 1.60 \frac{t}{1 \mathrm{y}} \frac{\rho(0)}{0.3 G e V c m^{-3}} \frac{m}{1 \mathrm{Kg}} \frac{\sqrt{\left\langle v^{2}\right\rangle}}{280 \mathrm{kms} s^{-1}} \frac{\sigma_{p, \chi^{0}}^{S}}{10^{-3} \mathrm{pb}} f_{\text {spin }}\left(A, Z, \mu_{r}(A)\right) \zeta_{\text {spin }} .
$$




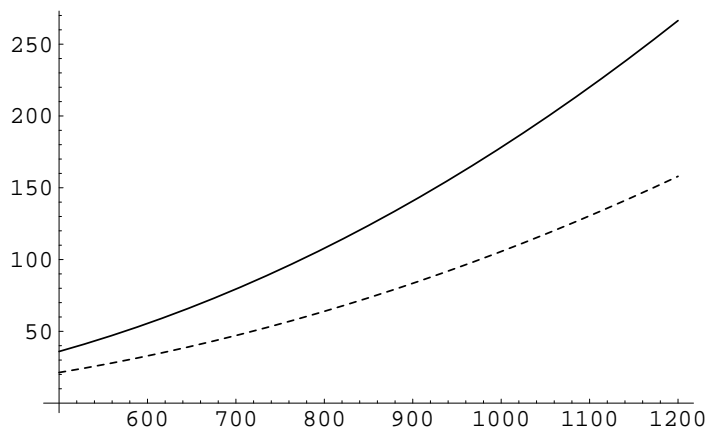

(a)

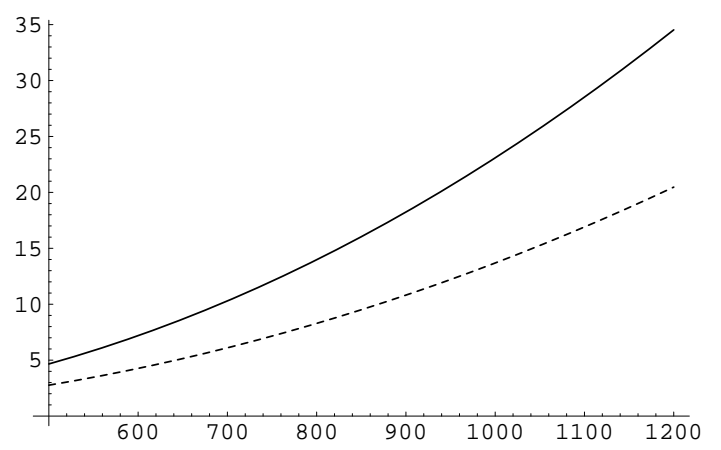

(c)

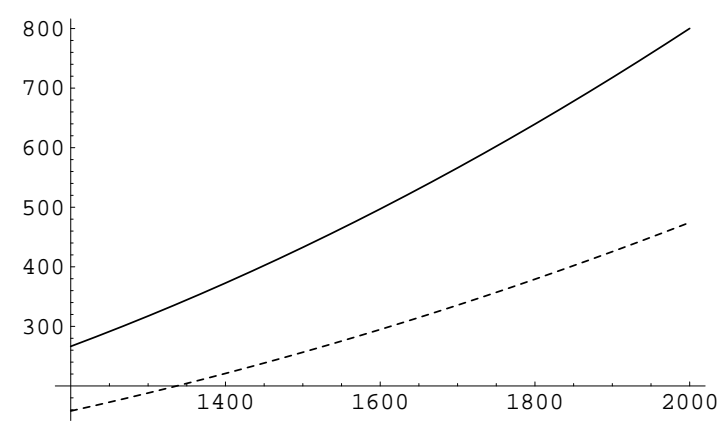

(b)

$$
m_{W I M P} \rightarrow \mathrm{GeV}
$$

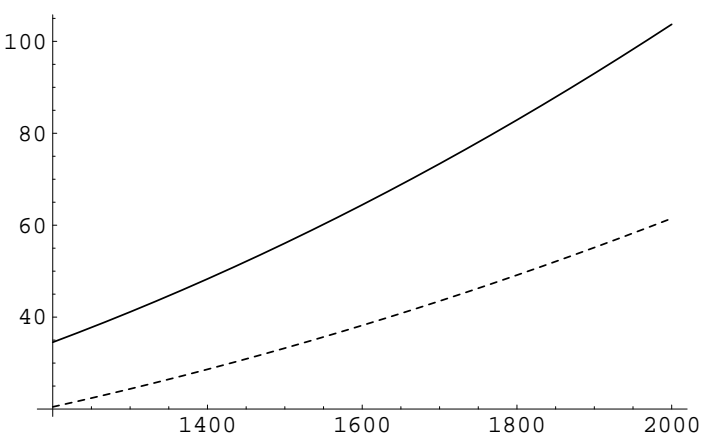

(d)

$$
m_{W I M P} \rightarrow \mathrm{GeV}
$$

FIG. 16: The same as in Fig. 15 when the WIMP is a K-K Majorana neutrino $\nu^{(1)}$. The coherent process is mediated by Higgs exchange, with $m_{h}=300 \mathrm{GeV}$ on the top and $500 \mathrm{GeV}$ at the bottom. The lighter Higgs mass is allowed by the data only for relatively light WIMPs. The heavier Higgs mass is allowed for all WIMPs. Clearly such heavy Higgs cannot occur in SUSY theories $\left(m_{h} \leq 120 \mathrm{GeV}\right)$.

Note that there is a different normalization, since due to the lack of coherence, the nucleon spin cross section must be larger to yield detectable results. In the above expression

$$
\zeta_{\text {spin }}=\frac{1}{3}\left(\Omega_{p}+\frac{a_{n}}{a_{p}} \Omega_{n}\right)^{2}
$$

with $a_{p}, a_{n}$ the proton and neutron spin amplitudes normalized so that [19] $\sigma_{p}=\left|a_{p}^{2}\right|$ and $\sigma_{n}=\left|a_{n}^{2}\right|$. $\Omega_{p}, \Omega_{n}$ are the nuclear spin matrix elements arising from the protons and neutrons respectively, normalized so that $\zeta=1$ for a single proton. The case of interest to us is when the WIMP is a K-K Majorana neutrino. In this case we have found that $a_{p}=-a_{n}$ and $\sigma_{p}=\sigma_{n}=8.010^{-3} \mathrm{pb}$. We will examine the following cases:

- The target ${ }^{19} \mathrm{~F}\left(\Omega_{p}=1.646, \Omega_{n}=0.30\right)$.

This light target is favored from the spin ME point of view [19, 23], but for heavy WIMPs is disfavored due to the small reduced mass. The parameter $t_{\text {spin }}$ is shown in Fig. 17. The obtained rates are shown in Fig. 18

- The target ${ }^{73} \mathrm{Ge}\left(\Omega_{p}=0.036, \Omega_{n}=1.040\right)$.

This medium mass target, favored for the coherent process as well, is characterized by large spin [19], 30]-31] induced rates. The parameter $t_{\text {spin }}$ is shown in Fig. 19] The obtained rates are shown in Fig. 20

- The target ${ }^{127} \mathrm{I}\left(\Omega_{p}=1.460, \Omega_{n}=0.355\right)$.

This medium mass target, with which the DAMA experiment [32, 33] claimed to have observed 


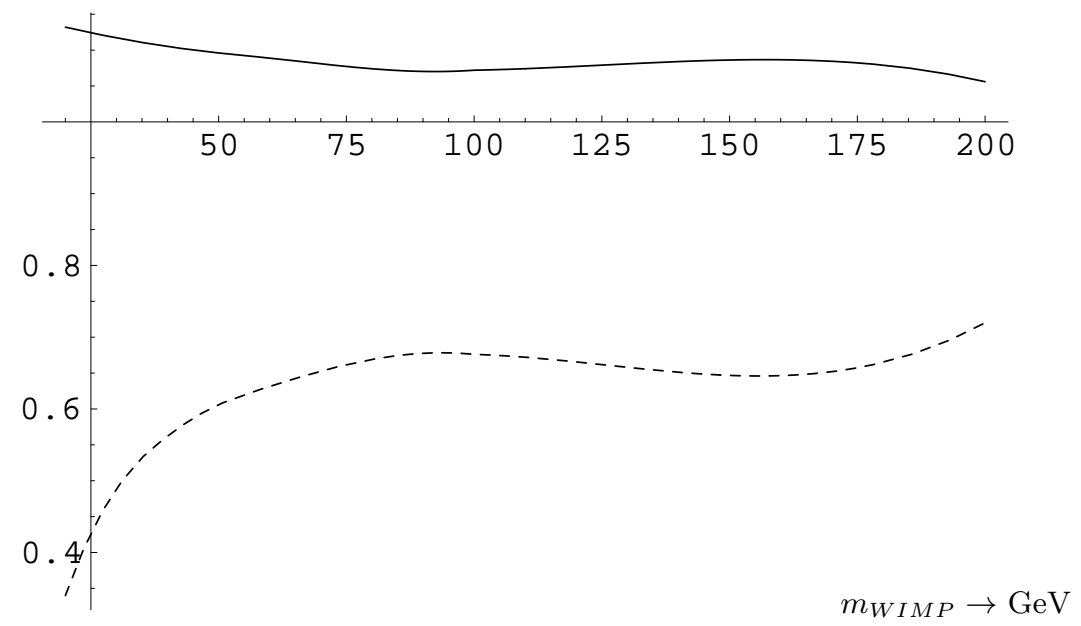

FIG. 17: The parameter $t_{\text {spin }}$ in the case of ${ }^{19} \mathrm{~F}$ as a function of the WIMP mass in GeV for zero threshold (continuous curve) and a threshold of $10 \mathrm{keV}$ (dotted curve). For higher WIMP masses $t_{\text {spin }}$ remains approximately constant.

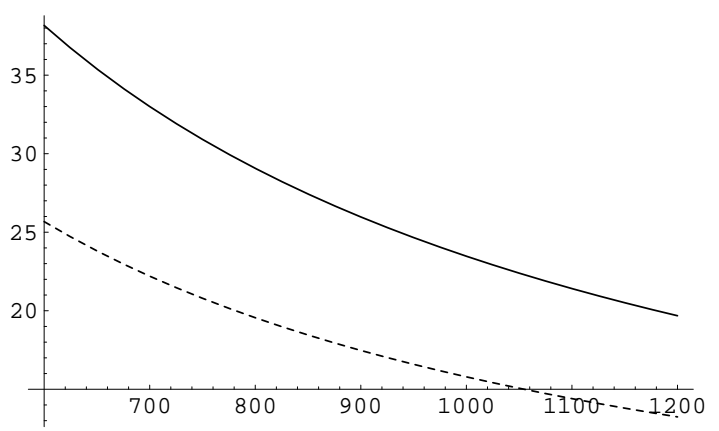

(a)

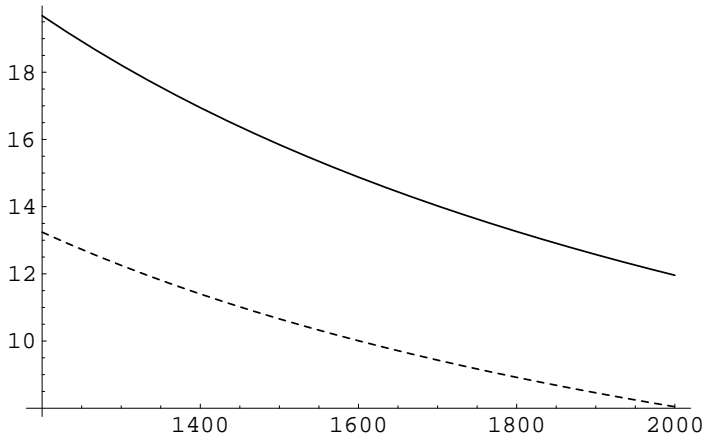

(b)

$$
m_{W I M P} \rightarrow \mathrm{GeV}
$$

FIG. 18: The spin event rate $R$ per year per $\mathrm{Kg}$ of target in the case of ${ }^{19} \mathrm{~F}$, in the cases the WIMP is a K-K majorana neutrino, plotted as a function of the WIMP mass in GeV for zero threshold (continuous curve) and a threshold of $10 \mathrm{keV}$ (dotted curve). Both figures show the same quantity except the WIMP masses range is different.

a signal, is favored for the spin contribution as well due to the large reduced mass, even though the spin ME is modest [30]-[31]. The parameter $t_{\text {spin }}$ is shown in Fig. 21. The obtained rates are shown in Fig. 22 We see that a K-K Majorana neutrino is not excluded by the data.

\section{DISCUSSION}

Even though the neutralino is the preferred WIMP candidate, in this work we concentrated on non-SUSY WIMPs. Extensions of the Standard Model, not motivated by some symmetry, involve many parameters and do not have much predictive power. So we will concentrate on K-K WIMPs, whereby the couplings involved are those of the Standard Model. Thus essentially one encounters only one unknown parameter, namely the WIMP mass.

¿From the results of the previous section, in connection with the K-K WIMPs as dark matter candidates, one can conclude the following: 


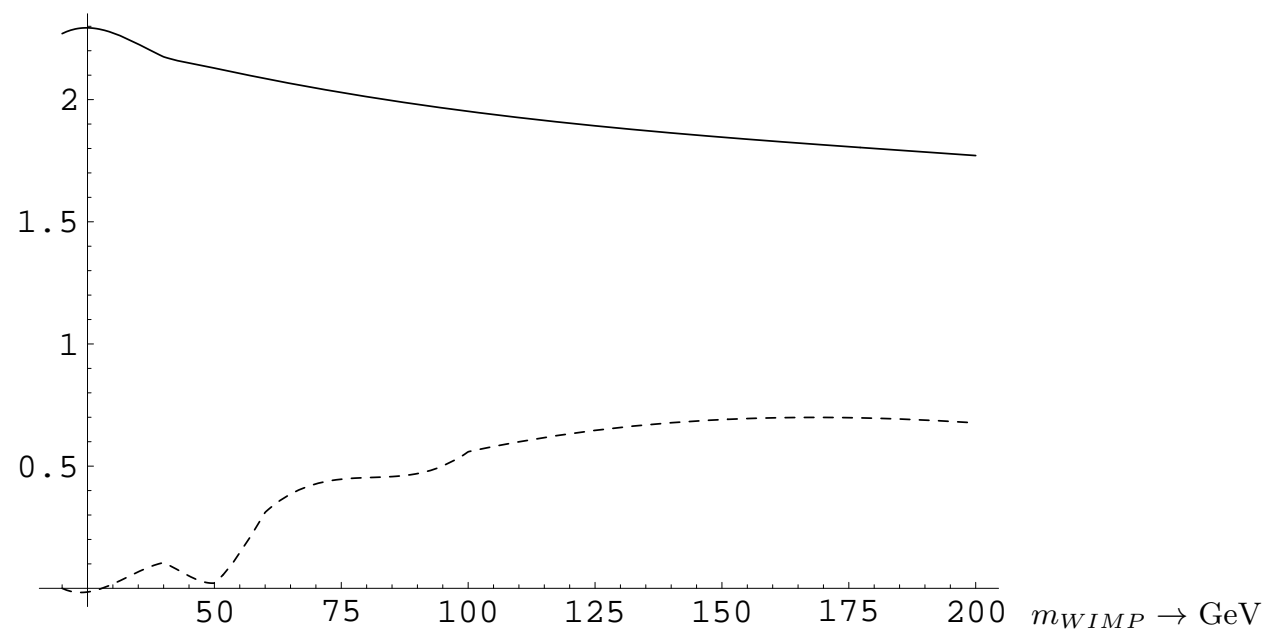

FIG. 19: The parameter $t_{\text {spin }}$ in the case of ${ }^{73}$ Ge. Otherwise the notation is the same as in Fig. 17

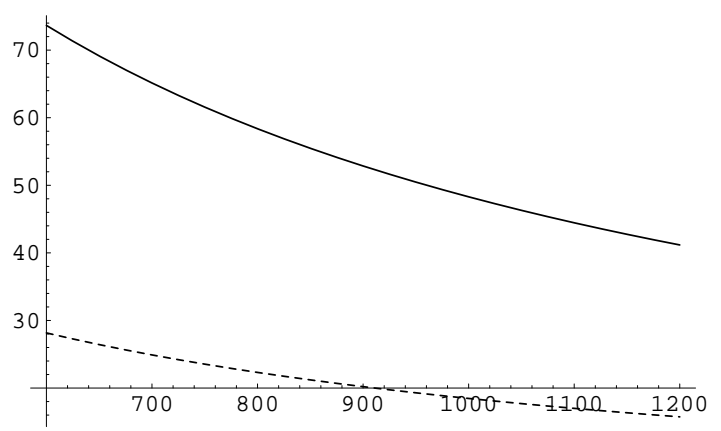

(a)

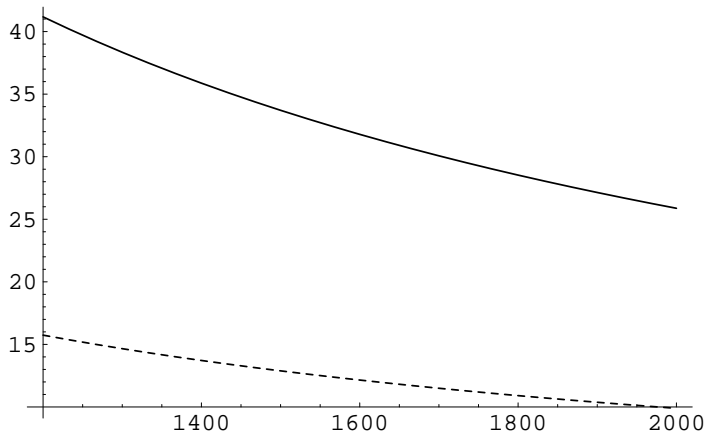

(b)

$$
m_{W I M P} \rightarrow \mathrm{GeV}
$$

FIG. 20: The same as in 18 in the case of the ${ }^{73}$ Ge target.

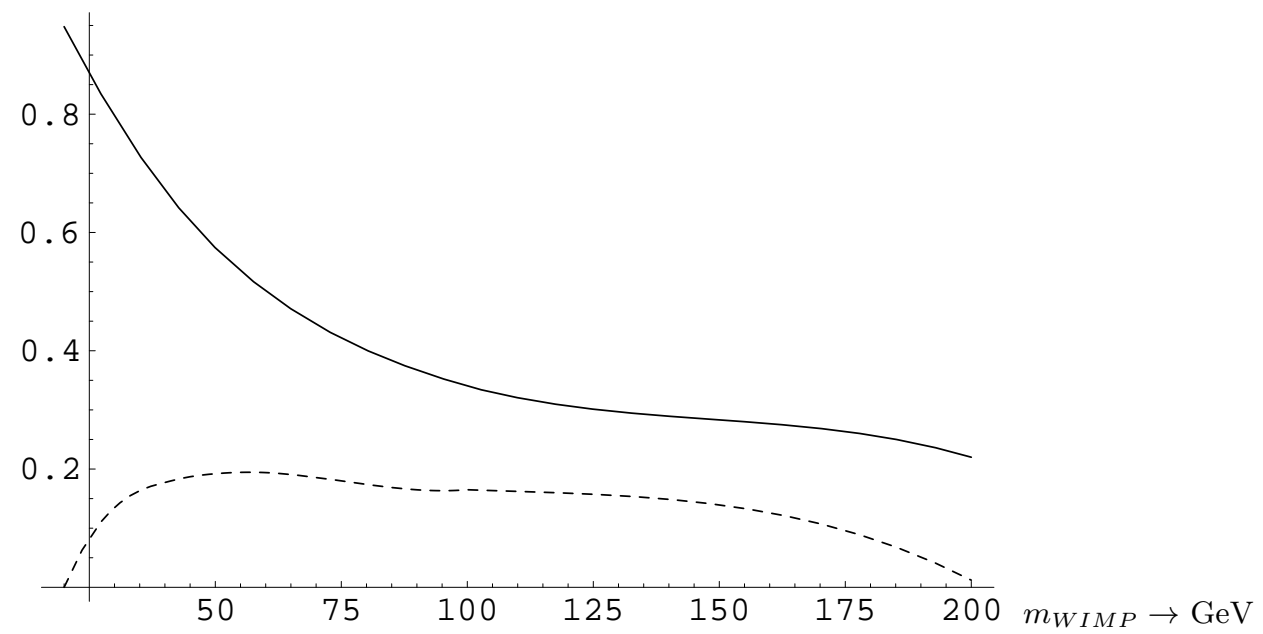

FIG. 21: The parameter $t_{\text {spin }}$ in the case of ${ }^{127} \mathrm{I}$. Otherwise the notation is the same as in Fig. 17. 


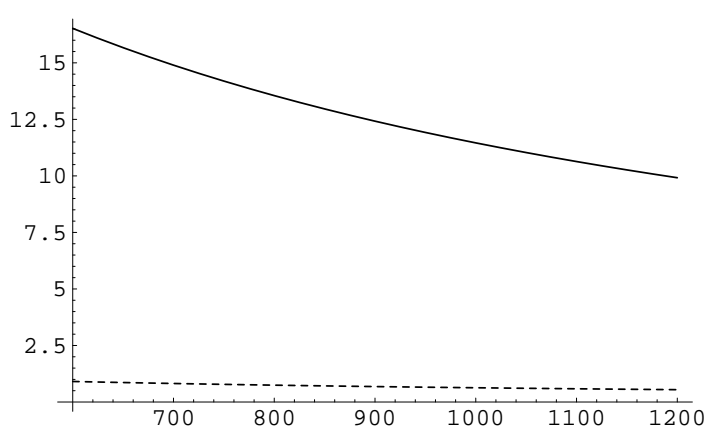

$m_{W I M P} \rightarrow \mathrm{GeV}$

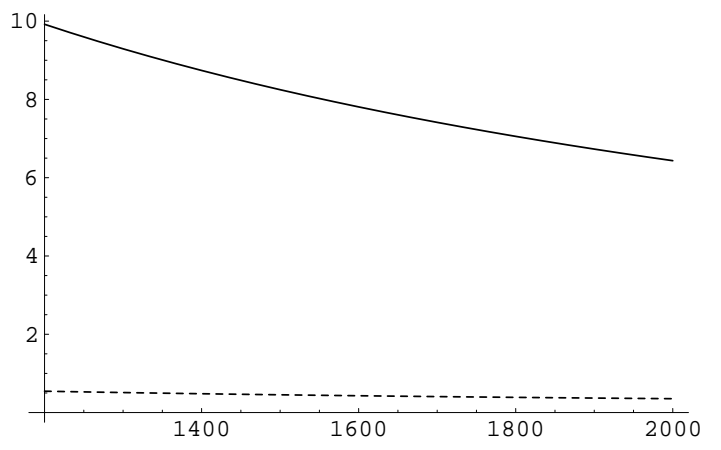

(a)

FIG. 22: The same as in 18 in the case of the ${ }^{127} \mathrm{I}$ target.

- The K-K neutrinos as CDM candidate.

In this case everything is under control, except of course, the fact that we do not know for sure whether the K-K neutrinos are Majorana or Dirac particles. Most authors expect them to be Dirac neutrinos (see, e.g. Servant [9]). In the case of Higgs contribution the nucleon cross section is the same both for Dirac and Majorana neutrinos. It is proportional to the $\left[m_{\nu^{(1)}}\right]^{2}$ and excludes the K-K neutrino as a viable WIMP candidate, unless the lightest Higgs is very heavy. In all other cases the WIMP mass enters via $\mu_{r}$, both explicitly and implicitly through the nuclear form factor. Anyway, since, essentially from cosmological requirements [1, 2], the $\mathrm{K}-\mathrm{K}$ neutrino mass is expected to be in the TeV region, $\mu_{r} \simeq A m_{p}$. So the cross section is independent of the WIMP mass. The number of WIMPs in our vicinity, for a given density, is inversely proportional to the WIMP mass. Thus the rate will scale as follows:

$$
R\left(m_{W I M P}\right)=R(A) \frac{A \mathrm{GeV}}{m_{W I M P}} .
$$

Large nucleon spin cross sections $\left(\sim 10^{-2}\right)$ pb are possible via Z-exchange. Thus Dirac neutrinos are excluded due to neutron coherence. No such coherence exists for Majorana neutrinos, so these can not be excluded from the data (16 events per year per $\mathrm{Kg}$ of target [34]). The precise predicted rates depend on nuclear physics assumptions.

- The K-K boson as CDM candidate.

For heavy WIMPs Eq. (53) holds in this case as well allowing larger nucleon cross-sections to be consistent with the data.

1. The unknown parameters of the theory are the masses of K-K quarks and gauge bosons as well as the mass of the neutral Higgs. All relevant couplings are under control.

2. In the spin independent mechanism the proton cross section is dominant. This is due to the RL+LR currents. This prediction can be consistent with the present data only away from the resonance and/or large K-K gauge boson masses. We should also take note of the fact that the event rate will be down by a factor $Z^{2} / A^{2}$ compared to the analysis of the neutralino case. This contribution ( $\mathrm{LR}+\mathrm{RL}$ ) is absent in the spin mode. It is also absent in the case of the neutron cross section.

3. Even in the other cases the proton cross sections are larger than those for the neutrons.

4. The results fall quite fast with increasing boson mass.

5. The obtained results, in particular those associated with the spin, are very sensitive functions of the mass difference $\Delta$ between the K-K quarks and the K-K bosons. 
6. For sufficiently small $\Delta$, the process involving the $\mathrm{K}-\mathrm{K}$ quarks is more important than the Higgs induced cross section. Away from the resonance the Higgs contribution becomes significant.

\section{Acknowledgments}

One of the authors (JDV) is indebted to Ignatios Antoniadis and Geraldine Servant for discussions during his visit at CERN. His work and this visit were supported by European Union under the contract MRTN-CT-2004-503369 as well as the program PYTHAGORAS-1. The latter is part of the Operational Program for Education and Initial Vocational Training of the Hellenic Ministry of Education under the 3rd Community Support Framework and the European Social Fund. The other two authors (V.K. Oikonomou and ChCM) acknowledge support by the co-funded European Union-European Social fund and National fund PYTHAGORAS -EPEAEK II.

[1] T. M. P. T. G. Servant, Nuc. Phys. B 650, 391 (2003).

[2] T. M. P. T. G. Servant, New Jour. Phys. 4, 99 (2002).

[3] S. D. I. Antoniadis, N. Arkani-Hamed, Phys. Lett. B 436, 267 (1998) ; arXiv:hep-ph/9804398.

[4] G. R. D. N. Arkani-Hamed, S. Dimopoulos, Phys. Rev. D 59, 086004 (1999) ; arXiv:hep-ph/9807344

[5] T. G. K. R. Dienes, E. Dudas, Nucl. Phys. B 47, 537 (1999) ; arXiv:hep-ph/9806292.

[6] B. A. D. T. Appelquist, H. C. Cheng, Phys. Rev. D 64, 035002 (2001) ; arXiv: hep-ph/0012100

[7] M. Q. I. Antoniadis, S. Dimopoulos, Nucl. Phys B 544, 503 (1999) ; arXiv: hep-ph/9810410.

[8] M. Q. I. Antoniadis, K. Benakli, Phys. Lett. B 331, 313 (1994) ; arXiv: hep-ph/9403290

[9] See, e.g., G. Servant, in Les Houches :Physics at TeV Colliders 2005" Beyond the Standard Model working group: summary report, B.C. Allanach (ed.), C. Grojean (ed.), P. Skands (ed.), al, section 25, p. 164; hep-ph/0602198

[10] S. Hanary et al: Astrophys. J. 545, L5 (2000); J.H.P Wu et al: Phys. Rev. Lett. 87, 251303 (2001); M.G. Santos et al: Phys. Rev. Lett. 88, 241302 (2002).

[11] P. D. Mauskopf et al: Astrophys. J. 536, L59 (2002); S. Mosi et al: Prog. Nuc.Part. Phys. 48, 243 (2002); S. B. Ruhl al, astro-ph/0212229 and references therein.

[12] N. W. Halverson et al: Astrophys. J. 568, 38 (2002) L. S. Sievers et al: astro-ph/0205287 and references therein.

[13] G. F. Smoot et al (COBE Collaboration), Astrophys. J. 396, L1 (1992).

[14] D. N. Spergel et al, Astrophys. J. Suppl. 148, 175 (2003).

[15] M. Tegmark et al, Phys.Rev. D 69, 103501 (2004).

[16] D.N. Spergel et al, Three-Year WMAP Results: Implications for Cosmology, astro-ph/0603449 L. Page et al, Three-Year WMAP Results: Polarization Analysis, astro-ph/0603450 G. Hinsaw et al, Three-Year WMAP Observations: Implications Temperature Analysis, astro-ph/0603451;

N Jarosik et al, Three-Year WMAP Observations: Beam Profiles, Data Processing, Radiometer Characterization and Systematic Error Limits, astro-ph/0603452.

[17] A. Djouadi and M. K. Drees, Phys. Lett. B 484, 183 (2000); S. Dawson, Nucl. Phys. B 359, 283 (1991); M. Spira it et al, Nucl. Phys. B453, 17 (1995).

[18] T. P. Cheng, Phys. Rev. D 38, 2869 (1988); H-Y. Cheng, Phys. Lett. B 219, 347 (1989).

[19] See, e.g., our recent review: J.D. Vergados, On the direct detection of dark matter- Exploring all the signatures of the neutralino-nucleus interaction, hep-ph/0601064 and references therein.

[20] The Strange Spin of the Nucleon, J. Ellis and M. Karliner, hep-ph/9501280.

[21] J. Ellis, K. A. Olive, Y. Santoso, and V. C. Spanos, Phys.Rev. D 70, 055005 (2004).

[22] A. Bottino et al., Phys. Lett B 402, 113 (1997).

R. Arnowitt. and P. Nath, Phys. Rev. Lett. 74, 4592 (1995); Phys. Rev. D 54, 2374 (1996); hep-ph/9902237.

V. A. Bednyakov, H.V. Klapdor-Kleingrothaus and S.G. Kovalenko, Phys. Lett. B 329, 5 (1994).

[23] P. C. Divari, T. S. Kosmas, J. D. Vergados, and L. D. Skouras, Phys. Rev. C 61, 054612 (2000).

[24] J. D. Vergados, J.Phys. G 30, 1127 (2004), 0406134. 
[25] G. S. G. Agashe, JCAP 0502, 002 (2005) ; arXiv:hep-ph/0411254.

[26] E. Ma, Mod.Phys.Lett. A 21, 1777 (2006); hep-ph/0605180.

[27] M. Cirelli, N. Forengo and A. Sturmia, Minimal Dark Matter, hep-ph/0512090 (to appear in Nuc. Phys.B 753).

[28] K. T. F. Sannino, Phys. Rev. D 71, 2005 (2005) ; S.V. Gudnason,C. Kouvaris and F Sannino, Dark Matter from New Technicolour Theories, hep-ph/0608055;.

[29] J. D. Vergados, Phys. Rev. D 57, 103003 (2003) hep-ph/0303231.

[30] M. T. Ressell et al., Phys. Rev. D 48, 5519 (1993); M.T. Ressell and D. J. Dean, Phys. Rev. C 56, 535 (1997).

[31] E. Homlund and M. Kortelainen and T. S. Kosmas and J. Suhonen and J. Toivanen, Phys. Lett B, 584,31 (2004); Phys. Atom. Nucl. 67, 1198 (2004).

[32] R. Bernabei et al, Phys. Lett. B 389, 757 (1996).

[33] R. Bernabei et al, Phys. Lett. B 424, 195 (1998).

[34] D. A. et al (CDMS collaboration), Phys. Rev. Let. 93, 211301 (2004). 